\title{
Pathogen-Associated Molecular Pattern-Induced TLR2 and TLR4 Activation Increases Keratinocyte Production of Inflammatory Mediators and is Inhibited by Phosphatidylglycerol
}

\author{
Vivek Choudhary, Shantelle Griffith, Xunsheng Chen, and Wendy B. Bollag
}

Charlie Norwood VA Medical Center, One Freedom Way, Augusta, Georgia (V.C., W.B.B.); and Departments of Physiology (V.C., S.G., X.C., W.B.B.) and Dermatology (W.B.B.), Medical College of Georgia at Augusta University, Augusta, Georgia

Received August 27, 2019; accepted February 25, 2020

\begin{abstract}
Skin serves not only as a protective barrier to microbial entry into the body but also as an immune organ. The outer layer, the epidermis, is composed predominantly of keratinocytes, which can be stimulated to produce proinflammatory mediators. Although some inflammation is useful to defend against infection, excessive or persistent inflammation can lead to the development of inflammatory skin diseases, such as psoriasis, a common skin disorder affecting approximately $2 \%$ of the US population. We have previously found that phosphatidylglycerol (PG) derived from soy can inhibit inflammation in a contact irritant ear edema mouse model. Here, we investigated the ability of soy $P G$ to inhibit inflammatory mediator expression in response to activators of the pattern recognition receptors, toll-like receptor2 (TLR2) and -4 (TLR4). We found that in epidermal keratinocytes, soy PG inhibited TLR2 and TLR4 activation and inflammatory mediator expression in response to a synthetic triacylated lipopeptide and lipopolysaccharide, respectively, as well as an endogenous danger-associated molecular pattern. However, at higher concentrations, soy PG alone enhanced the expression of some proinflammatory cytokines, suggesting a narrow
\end{abstract}

therapeutic window for this lipid. Dioleoylphosphatidylglycerol (DOPG), but not dioleoylphosphatidylcholine, exerted a similar inhibitory effect, completely blocking keratinocyte inflammatory mediator expression induced by TLR2 and TLR4 activators as well as $\mathrm{NF} \kappa \mathrm{B}$ activation in a macrophage cell line (RAW264.7); however, DOPG was not itself proinflammatory even at high concentrations. Furthermore, DOPG had no effect on $\mathrm{NF}_{\kappa} \mathrm{B}$ activation in response to a TLR7/8 agonist. Our results suggest that DOPG could be used to inhibit excessive skin inflammation.

\section{SIGNIFICANCE STATEMENT}

Although inflammation is beneficial for clearing an infection, in some cases, the infection can be excessive and/or become chronic, thereby resulting in considerable tissue damage and pathological conditions. We show here that the phospholipid phosphatidylglycerol can inhibit the activation of toll-like receptors 2 and 4 of the innate immune system as well as the downstream inflammatory mediator expression in response to microbial component-mimicking agents in epidermal keratinocytes that form the physical barrier of the skin.

\section{Introduction}

Skin serves as a protective barrier to microorganisms and as such, plays an important role as a component of the innate immune system (Pivarcsi et al., 2003). The outer layer, the epidermis, is composed predominantly of keratinocytes, the proliferation and differentiation of which are precisely regulated to properly form the epidermal mechanical and waterpermeability barrier (Yuspa et al., 1990; Goldsmith, 1991). Dysregulation of this programmed pattern of growth and

This research was supported in part by Veterans Affairs Merit Award [CX001357]. S.G. was supported by a Medical College of Georgia Medical Scholars Program Award. W.B.B. was supported in part by a Veterans Affairs Research Career Scientist Award. The contents of this article do not represent the official views of the Department of Veterans Affairs or the United States Government.

https://doi.org/10.1124/mol.119.118166. maturation can lead to an abnormal barrier, which characterizes a variety of skin diseases, including psoriasis (Langley, 2005). Psoriasis is an immune-mediated skin disease, exhibiting hyperproliferation and abnormal differentiation of keratinocytes in addition to inflammation. Patients with psoriasis report a reduction in their quality of life similar to that described by patients with more life-threatening illnesses (Rapp et al., 1999; Stern et al., 2004). Accumulating evidence points to a possible involvement of the innate immune system in inflammatory skin diseases like psoriasis [reviewed in Schön (2019)].

We previously found that phosphatidylglycerol (PG) can be generated by the combined action of the glycerol channel aquaporin-3 (AQP3) and the lipid-metabolizing enzyme phospholipase D2 (PLD2) (Zheng and Bollinger Bollag, 2003; Bollag et al., 2007; Xie et al., 2014). These two proteins are

ABBREVIATIONS: AQP3, aquaporin-3; DAMP, danger- or damage-associated molecular pattern; DOPC, dioleoylphosphatidylcholine; DOPG, dioleoylphosphatidylglycerol; GAPDH, glyceraldehyde-3-phosphate dehydrogenase; IL, interleukin; LPS, lipopolysaccharide; NF $\kappa$ B, nuclear factor $\kappa \mathrm{B} ; \mathrm{Pam}_{3} \mathrm{CSK}_{4}$, tripalmitoylated cysteine-serine-lysine-lysine-lysine-lysine, a synthetic triacylated peptide TLR1/2 agonist; PAMP, pathogenassociated molecular pattern; PG, phosphatidylglycerol; PLD2, phospholipase D2; RT-PCR, real-time polymerase chain reaction; SEAP, secreted embryonic alkaline phosphatase; TLR, toll-like receptors; TNF, tumor necrosis factor. 
colocalized in epidermal keratinocytes (Zheng and Bollinger Bollag, 2003), with PLD able to convert the glycerol transported by AQP3 to PG. PG levels were found to increase upon keratinocyte differentiation in response to medium with elevated calcium concentrations, with maximal levels reached at a calcium concentration that is optimal for inducing early keratinocyte differentiation (Zheng et al., 2003), suggesting a potential role in this process. Furthermore, manipulations to alter the function of this novel AQP3/PLD2 signaling pathway affected keratinocyte differentiation (Bollag et al., 2007; Choudhary et al., 2015). Importantly, egg-derived PG normalizes keratinocyte function, inhibiting the proliferation of keratinocytes that are rapidly dividing and stimulating growth in cells that are dividing slowly. The effect seems to be specific to PG because a similar phospholipid phosphatidylpropanol did not significantly affect keratinocyte proliferation (Bollag et al., 2007). We subsequently showed that the fatty acids composing $\mathrm{PG}$ also played an important role in the effect of different PG species on keratinocyte proliferation. Thus, PG species containing polyunsaturated fatty acids are more effective at inhibiting, and those with saturated or monounsaturated fatty acids are better able to stimulate, keratinocyte growth (Xie et al., 2014). Soy PG possesses a large percentage of polyunsaturated fatty acids, with particular efficacy to inhibit keratinocyte proliferation (Xie et al., 2014), suggesting that it might be used to suppress the keratinocyte hyperproliferation characteristic of psoriasis. Indeed, we showed that soy PG inhibits inflammation and mouse ear swelling induced by the contact irritant 12-O-tetradecanoylphorbol 13-acetate in vivo (Xie et al., 2018). However, the mechanism by which soy PG functions to suppress inflammation is unclear.

Toll-like receptors (TLRs) are pattern-recognition receptors, which upon activation can stimulate the innate immune system. In response to microbial components [so-called pathogen-associated molecular patterns (PAMPs)] or endogenous molecules released by threatened or injured cells [dangeror damage-associated molecular patterns (DAMPs)], TLRs are known to initiate a signaling cascade that results in downstream activation of nuclear factor $\kappa \mathrm{B}\left(\mathrm{NF}_{\kappa} \mathrm{B}\right)$. $\mathrm{NF}_{\kappa} \mathrm{B}$ activation occurs through adaptor proteins such as myeloid differentiation primary response gene 88 and TIR-domain-containing adapter inducing interferon- $\beta$; E3 ligases such as TNF receptor-associated factor-6; and kinases such as IL1 receptor-associated kinases 1, 2, and 4 , receptor-interacting protein-1, and TGF $\beta$-activating kinase-1 [reviewed in Adelaja and Hoffmann (2019)]. The cascade results in TGF $\beta$-activating kinase- $1-$ mediated activation of inhibitor of $\kappa \mathrm{B}$ kinase to phosphorylate and induce the degradation of inhibitor of $\kappa \mathrm{B}$, resulting in $\mathrm{NF} \kappa \mathrm{B}$ nuclear translocation and activation (Adelaja and Hoffmann, 2019). The transcription factor $\mathrm{NF} \kappa \mathrm{B}$ is the principal regulator of inflammatory gene expression (Adelaja and Hoffmann, 2019).

In a previous study, we found that in keratinocytes and a macrophage cell line, dioleoylphosphatidylglycerol (DOPG) inhibits inflammatory mediator production in response to TLR2 and TLR4 activation by the antimicrobial peptide S100A9 (Choudhary et al., 2019). In this study, we were also able to show an inhibition of skin inflammation in vivo in an imiquimod-induced mouse model of psoriasis (Choudhary et al., 2019). Whether soy PG and DOPG inhibit TLR activation in keratinocytes stimulated by PAMPs is unknown. Also unclear is whether $\mathrm{PG}$ can inhibit TLR-mediated $\mathrm{NF} \kappa \mathrm{B}$ activation in response to PAMPs to exert its anti-inflammatory activity.

To test the idea that soy PG might affect sterile and infectious inflammation through effects on TLR2 and TLR4 activation, keratinocytes and the macrophage cell line RAW264.7 were treated with TLR2 and TLR4 agonists in the presence and absence of soy PG, and inflammatory mediator expression was monitored. Our results provide evidence for the anti-inflammatory effects of PG, suggesting the potential use of this phospholipid to treat inflammatory skin disorders such as psoriasis.

\section{Materials and Methods}

Materials. Recombinant S100A9 was purchased from R\&D Systems (Minneapolis, MN); primary antibodies recognizing p65-NF $\kappa$ B phosphorylated on serine $536(\mathrm{pNF} \kappa \mathrm{B}$; catalog number 3033$)$ or total p65-NF $\kappa \mathrm{B}$ (catalog number 6956) were from Cell Signaling Technology (Danvers, MA); HEK-Blue hTLR2 (catalog number hkb-htlr2), HEK-Blue hTLR4 (catalog number hkb-htlr4), and Resiquimod were from InvivoGen (San Diego, CA); and Graphpad Prism was from Graphpad (La Jolla, CA). Pam ${ }_{3} \mathrm{CSK}_{4}$ and LPS were purchased from Sigma-Aldrich (St. Louis, MO).

Keratinocyte Preparation and Cell Culture. Primary cultures of mouse epidermal keratinocytes were prepared from 1- to- 3-day-old Institute of Cancer Research CD-1 outbred neonatal mice of both sexes as described in detail in Bollag et al. (1993) and Griner et al. (1999). All procedures were approved by the Institutional Animal Care and Use Committees of Augusta University or the Charlie Norwood VA Medical Center. RAW264.7 cells, purchased from American Type Culture Collection (Manassas, VA), were kindly provided by Drs. Qing Zhong and Carlos Isales (Augusta University) and were cultured in Dulbecco's modified Eagle's medium containing 10\% fetal bovine serum and $1 \%$ penicillin and streptomycin.

Quantitative Real-Time Polymerase Chain Reaction. Keratinocytes were treated with or without a synthetic triacylated lipoprotein $\left(\mathrm{Pam}_{3} \mathrm{CSK}_{4}\right)$, a TLR1/TLR2 agonist, lipopolysaccharide, a TLR4 agonist, or recombinant human S100A9 (as in Choudhary et al., 2019) for 2 hours in the presence and absence of different concentrations of soy PG or DOPG as indicated. Cells were harvested and RNA isolated using PerfectPure RNA tissue kits (5 PRIME, Inc., Gaithersburg, MD) as per the manufacturer's protocol. Following verification of RNA integrity using a Nanodrop instrument (NanoDrop Technologies, Wilmington, DE), RNA was reverse-transcribed to cDNA with iScript cDNA synthesis kits (Bio-Rad Laboratories, Hercules, CA) and analyzed by real-time polymerase chain reaction (RT-PCR) using Taqman primer/probe sets from Applied Biosystems (Life Technologies, Grand Island, NY). Reactions were performed using Fast Reagent PCR Master Mix (Applied Biosystems) and a StepOnePlus Real-Time PCR System (Applied Biosystems) as instructed by the manufacturer. Expression of genes of interest was determined using the $\delta-\delta \mathrm{Ct}$ method normalized to the expression of glyceraldehyde-3-phosphate dehydrogenase (GAPDH) as the endogenous housekeeping gene and expressed as the fold increase relative to the appropriate control. For each individual experiment, the maximum fold increase was then identified and set to $100 \%$ with all values expressed relative to this maximal value. The results were analyzed in this way to account for differences in the magnitude of the response (i.e., the fold change) in experiments performed over time by normalizing the responses between $0 \%$ and $100 \%$.

TLR2 or TLR4 Activation in the HEK-Blue-hTLR2 Reporter Cell Line. TLR2 or TLR4 activation was determined in the HEKBlue-hTLR2 or HEK-Blue-hTLR4 reporter cell lines according to the supplier's instructions. These HEK293 cells are engineered to express human TLR2 or TLR4 together with the MD2 and CD14 coreceptors. They also express secreted embryonic alkaline phosphatase (SEAP) 
under the control of a promoter with binding sites for the transcription factors $\mathrm{NF}_{\kappa} \mathrm{B}$ and activator protein-1. $\mathrm{Pam}_{3} \mathrm{CSK}_{4}$ or LPS with or without DOPG or dioleoylphosphatidylcholine (DOPC) (in $20 \mu \mathrm{l}$ ) was added to a 96-well cell culture plate. HEK-Blue hTLR2 or hTLR4 cells at 70\%-80\% confluence were detached in PBS. Cells resuspended in HEK-Blue Detection medium [at a density of approximately 280,000 (TLR2) or 140,000 (TLR4) cells $/ \mathrm{ml}$ in $180 \mu \mathrm{l}$ ] were added to each well with or without phospholipid and incubated at $37^{\circ} \mathrm{C}$ and $5 \% \mathrm{CO}_{2}$ for 18 or 24 hours as indicated. SEAP activity was measured as absorbance at $620 \mathrm{~nm}$ using a BIO-TEK Synergy HT microplate reader with Gen5 analysis software.

Western Analysis and Immunocytochemistry. Immunocytochemical analysis of $\mathrm{NF}_{\kappa} \mathrm{B}$ nuclear translocation and Western analysis of $\mathrm{NF}_{\kappa} \mathrm{B}$ phosphorylation was performed as previously described (Choudhary et al., 2019).

Statistical Analyses. Statistical analyses were performed using ANOVA followed by Tukey's post hoc tests, as performed by GraphPad Prism, with significance established at $P \leq 0.05$.

\section{Results}

Effects of TLR2 Activation with a Synthetic Triacylated Lipoprotein, Pam $_{3} \mathrm{CSK}_{4}$, a TLR1/2 Agonist, on Keratinocyte Expression of Inflammatory Mediators. We have previously shown that S100A9 and $\beta$-defensin- 2 can activate TLR2 (Choudhary et al., 2019), presumably by acting as DAMPs. To determine if PAMPs can also induce keratinocyte expression of inflammatory mediators, we first examined the effect of TLR2 stimulation with the synthetic triacylated lipoprotein TLR1/2 agonist $\mathrm{Pam}_{3} \mathrm{CSK}_{4}$, which mimics the acylated amino terminus of bacterial lipoproteins, on the mRNA levels of keratinocyte inflammatory mediators. $\mathrm{Pam}_{3} \mathrm{CSK}_{4}$ dosedependently stimulated the expression of IL $1 \alpha$ and $\mathrm{TNF} \alpha$. On the other hand, IL6 was induced to a maximal plateau by the lowest $\mathrm{Pam}_{3} \mathrm{CSK}_{4}$ concentration, and IL1 $\beta$ was induced to the greatest extent by the lowest concentration, with higher doses actually producing a lesser response (Fig. 1). Furthermore, we examined the time course of the effects of $\mathrm{Pam}_{3} \mathrm{CSK}_{4}$ on mRNA levels of these inflammatory mediators and found a timedependent ability of $\mathrm{Pam}_{3} \mathrm{CSK}_{4}$ to increase the expression of these cytokines, with maximal expression of IL1 $\alpha$ and $\mathrm{TNF} \alpha$ at 2 hours and of IL1 $\beta$ and IL6 at 6 hours (Fig. 2). Interestingly, $\mathrm{Pam}_{3} \mathrm{CSK}_{4}$ also induced the expression of S100A9 (Fig. 2), indicating that stimulation of TLR2 promotes the expression of a TLR2 agonist (S100A9) and suggesting the possibility that a feed-forward loop might be established to promote chronic skin inflammation.

Effects of Soy PG on the Keratinocyte Expression of Inflammatory Mediators upon Stimulation with $\mathbf{P a m}_{\mathbf{3}} \mathbf{C S K}_{4}$. We previously showed that soy PG inhibits inflammation in a contact irritant ear edema mouse model in vivo (Xie et al., 2018). To determine whether soy PG's effect could result from an inhibition of TLR2 activation to block keratinocyte expression of inflammatory mediators, we treated primary mouse epidermal keratinocytes with 0,10 , and $50 \mu \mathrm{g} / \mathrm{ml}$ soy PG in the presence or absence of $\mathrm{Pam}_{3} \mathrm{CSK}_{4}$. As shown in Fig. 3, we found that $\mathrm{Pam}_{3} \mathrm{CSK}_{4}$ increased the mRNA expression of the
A

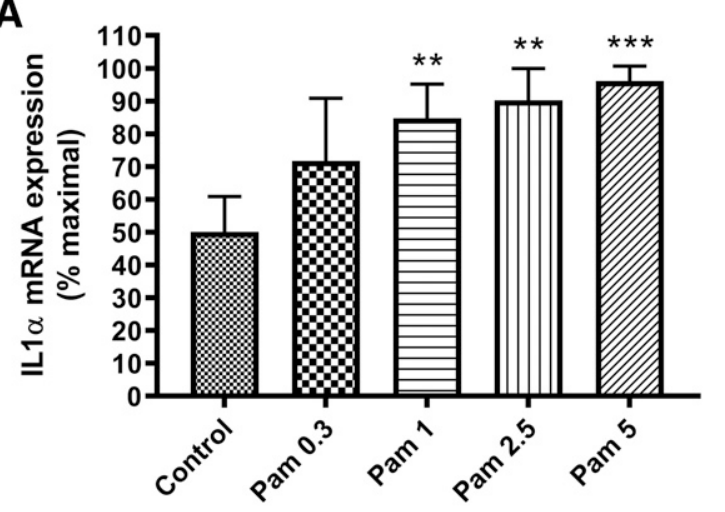

C

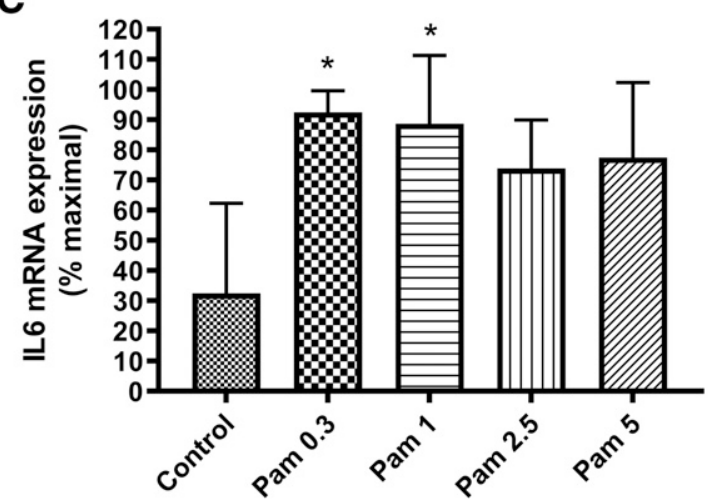

B

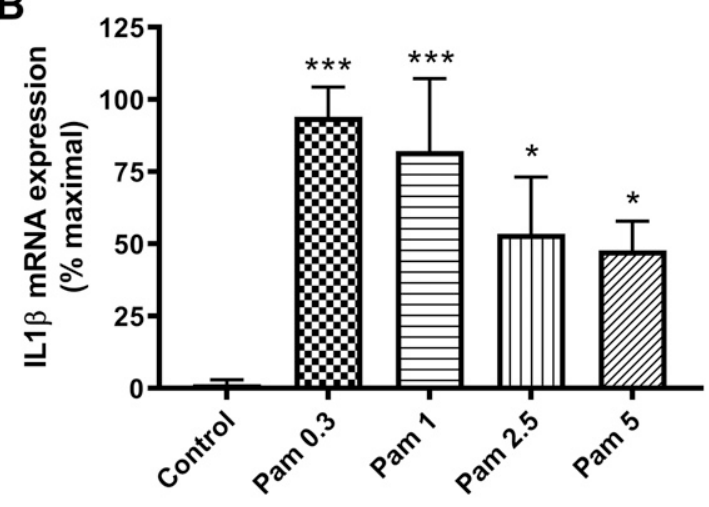

D

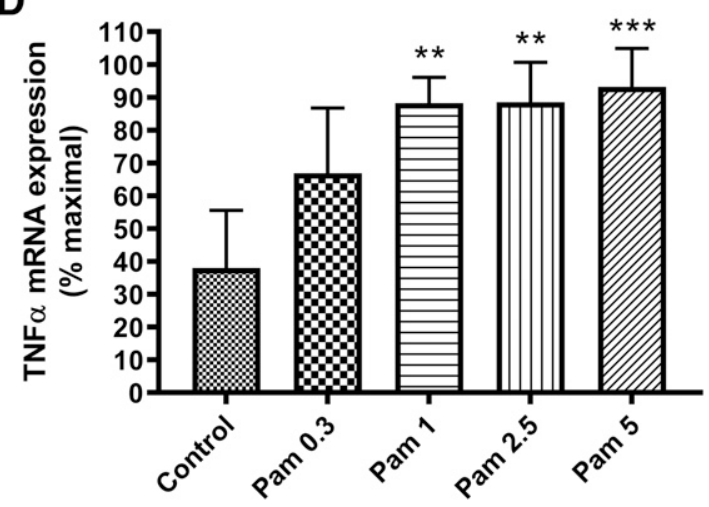

Fig. 1. The TLR1/2 agonist, $\mathrm{Pam}_{3} \mathrm{CSK}_{4}$, induced keratinocyte expression of inflammatory mediators in a dose-dependent manner. Keratinocytes were treated with various concentrations of the synthetic triacylated lipoprotein $\mathrm{Pam}_{3} \mathrm{CSK}_{4}(\mathrm{Pam})$ for 2 hours as indicated (in microgram per milliliter). RNA was then isolated, and the expression of the inflammatory mediators (A) IL1 $\alpha$, (B) IL1 $\beta$, (C) IL6, and (D) TNF $\alpha$ was monitored by quantitative RT-PCR with GAPDH used as the housekeeping gene. Results represent the means \pm S.D. of three separate experiments; $* P<0.05 ; * * P<0.01 ; * * * P<0.001$ vs. the zero concentration (Control). 
A

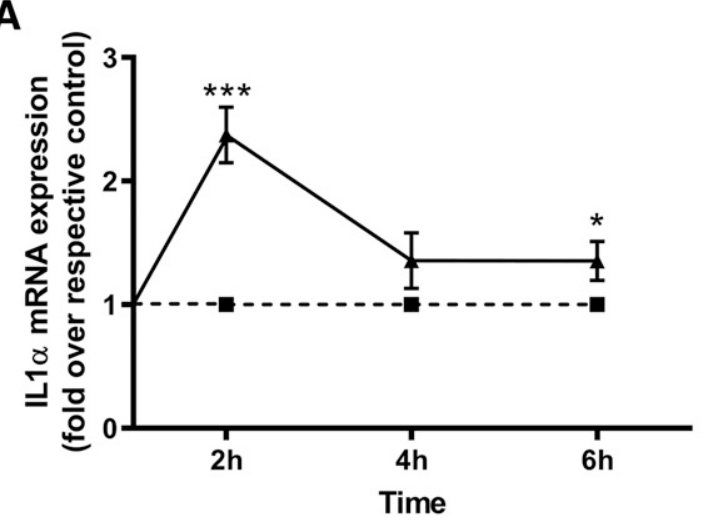

C

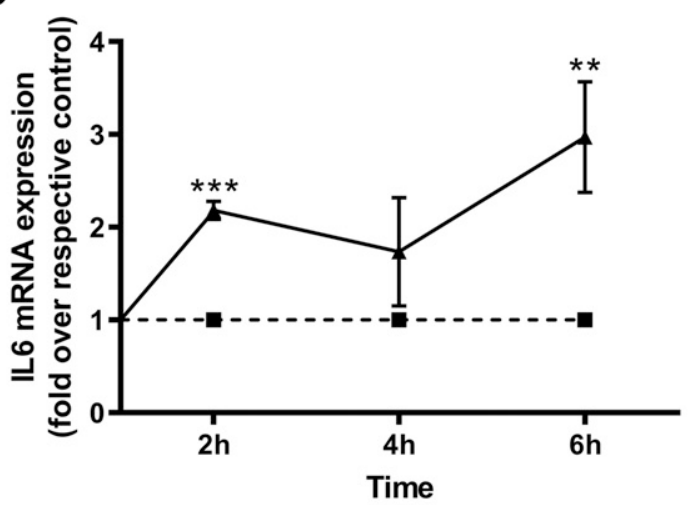

$\mathbf{E}$

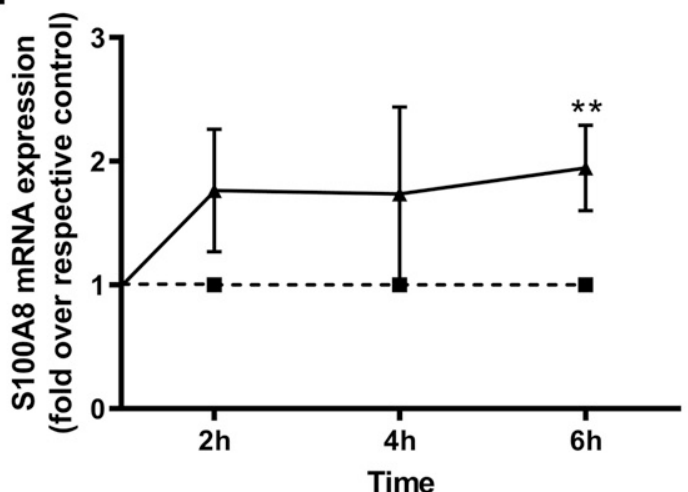

B

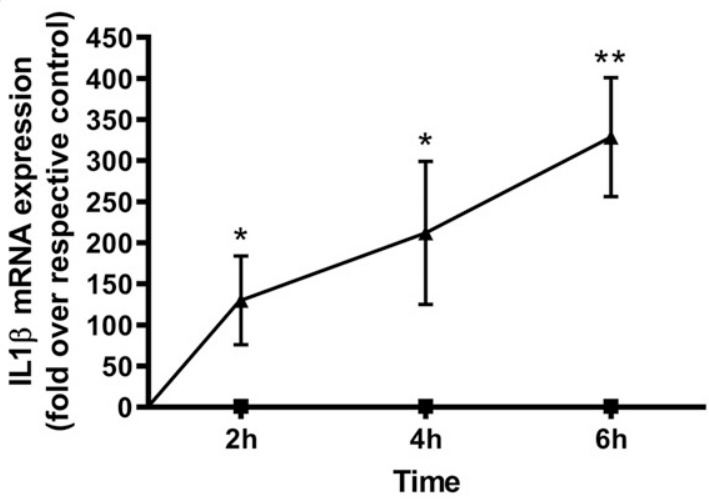

D

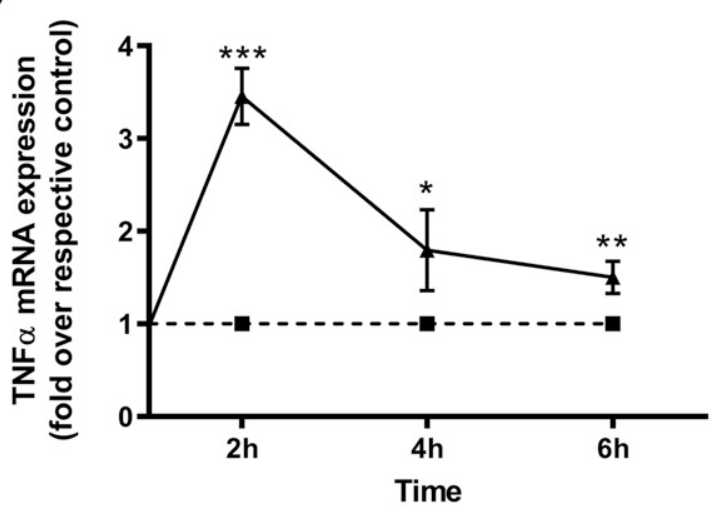

$\mathbf{F}$

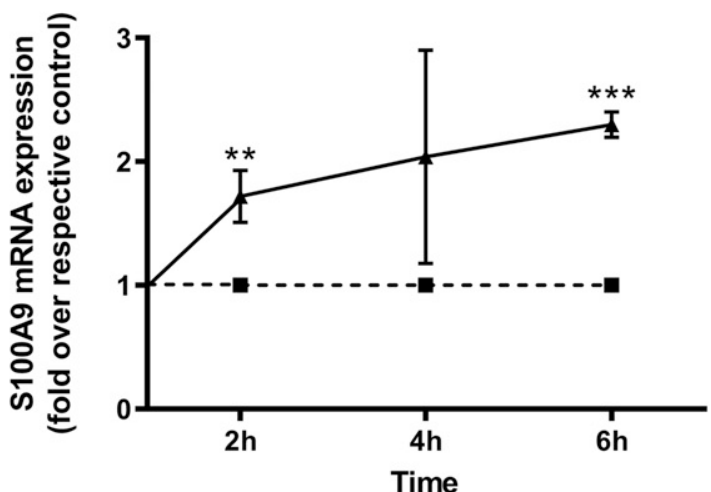

Fig. 2. $\mathrm{Pam}_{3} \mathrm{CSK}_{4}$ induced keratinocyte expression of inflammatory mediators in a time-dependent manner. Keratinocytes were treated with vehicle or $2.5 \mu \mathrm{g} / \mathrm{ml} \mathrm{Pam}_{3} \mathrm{CSK}_{4}(\mathrm{Pam})$ for 2, 4, and 6 hours. RNA was then isolated, and the expression of the inflammatory mediators (A) IL1 $\alpha$, (B) IL1 $\beta$, (C) IL6, and (D) TNF $\alpha$ as well as (E) S100A8 and (F) S100A9 was monitored by quantitative RT-PCR with GAPDH used as the housekeeping gene. Results represent the means \pm S.D. of three separate experiments; Pam $_{3} \mathrm{CSK}_{4}$-treated values were analyzed at each time point using one-sample $t$ tests vs. a hypothetical mean of 1.0 (to which the vehicle-treated control value was set), as performed by GraphPad Instat, with $* P<0.05$; $* * P<0.01$; $* * * P<0.001$ vs. the control at that time point.

inflammatory mediators $\mathrm{TNF} \alpha$, IL6, IL $1 \alpha$, and IL1 $\beta$ at $2.5 \mu \mathrm{g} / \mathrm{ml} \mathrm{Pam}_{3} \mathrm{CSK}_{4}$. Importantly, pretreatment with 10 and $50 \mu \mathrm{g} / \mathrm{ml}$ concentrations of soy PG blocked the increase in $\mathrm{TNF} \alpha$ and IL6; $10 \mu \mathrm{g} / \mathrm{ml}$ soy PG also returned the $\mathrm{Pam}_{3} \mathrm{CSK}_{4^{-}}$ induced increase in IL1 $\alpha$ to a level that was not statistically significantly different from the control value. However, soy PG had no effect on $\mathrm{Pam}_{3} \mathrm{CSK}_{4}$-elicited IL1 $\beta$ expression. This result suggests that soy PG can inhibit some of the inflammatory mediators that are expressed upon the activation of TLR1/2, which may be activated in psoriasis as a result of the upregulation of agonists such as S100A8 and S100A9 (Choudhary et al., 2019). In addition, it is possible that the expression of other (unexamined) cytokines may be similarly affected, as we have observed previously (Choudhary et al., 2019).

Effects of Soy PG on the Keratinocyte Expression of Inflammatory Mediators upon Stimulation with LPS. To determine whether soy PG can also inhibit TLR4 activation in keratinocytes, thereby blocking keratinocyte expression of inflammatory mediators, we treated primary mouse epidermal keratinocytes with 0,10 , and $50 \mu \mathrm{g} / \mathrm{ml}$ soy PG in the presence or absence of LPS. As shown in Fig. 4, we found that LPS increased the mRNA expression of the inflammatory mediators IL $1 \alpha$, IL $1 \beta$, and TNF $\alpha$ as well as the receptors for $\mathrm{Pam}_{3} \mathrm{CSK}_{4}$, TLR1, and TLR2. Pretreatment with 10 and 
A

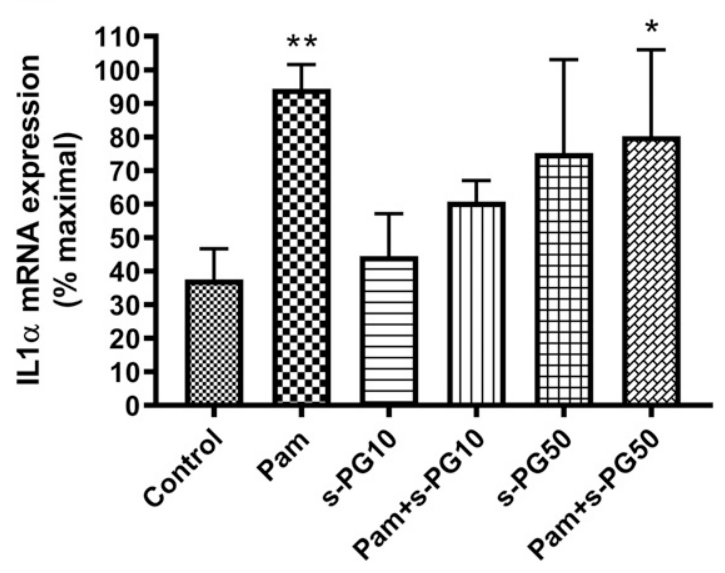

C

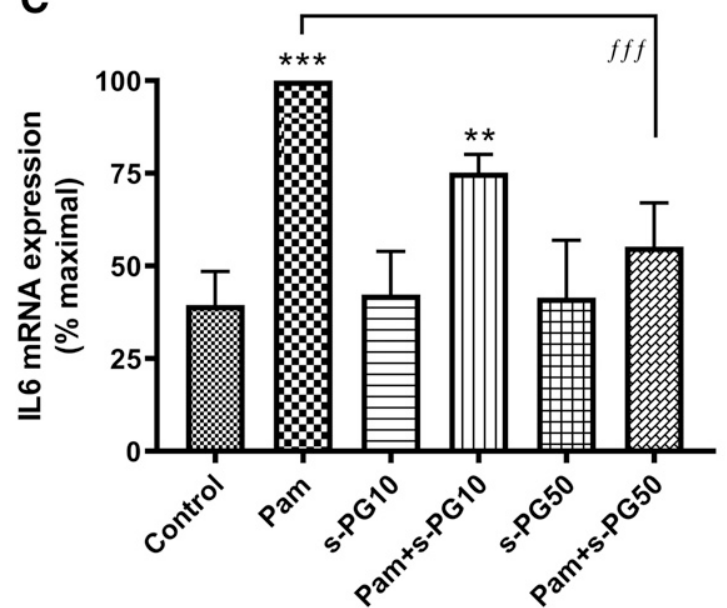

B

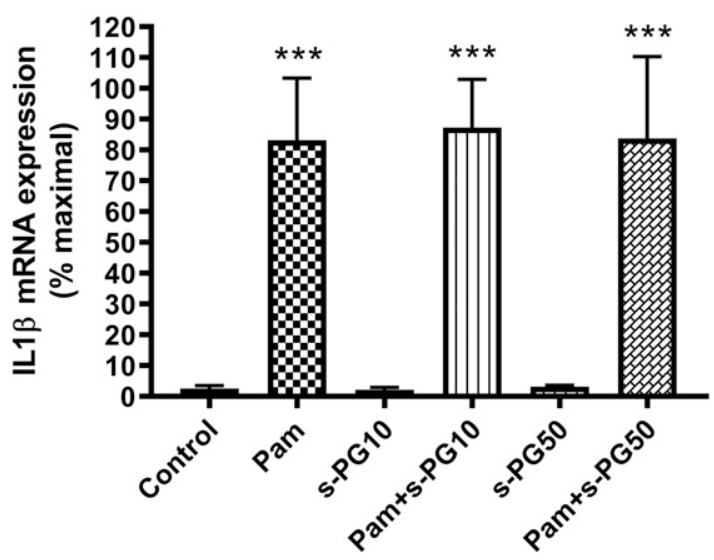

D

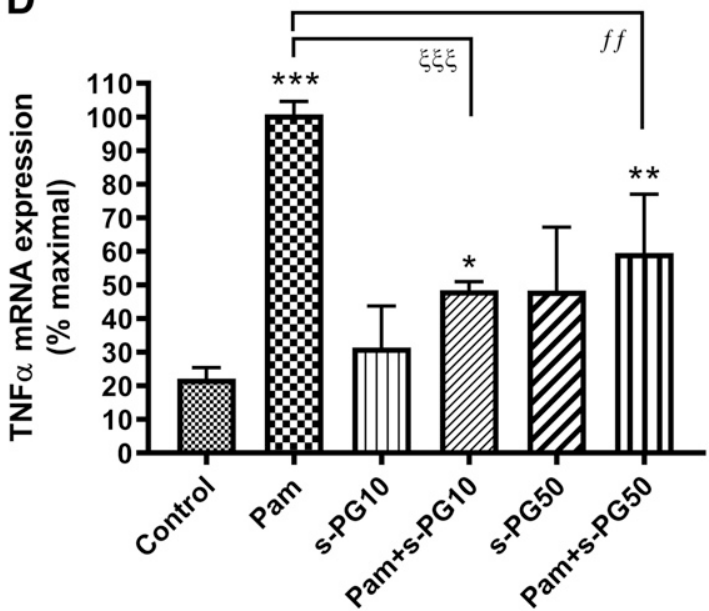

Fig. 3. Soy PG inhibited keratinocyte inflammatory mediator expression in response to $\mathrm{Pam}_{3} \mathrm{CSK}_{4}$. Keratinocytes were treated with 0 , 1 , and $2.5 \mu \mathrm{g} / \mathrm{ml}$ $\mathrm{Pam}_{3} \mathrm{CSK}_{4}(\mathrm{Pam})$ in the presence or absence of 10 or $50 \mu \mathrm{g} / \mathrm{ml}$ soy PG (s-PG) for 2 hours. RNA was then isolated, and the expression of the inflammatory mediators (A) IL1 $\alpha$, (B) IL1 $\beta$, (C) IL6, and (D) TNF $\alpha$ was monitored by quantitative RT-PCR with GAPDH used as the housekeeping gene. Results represent the means \pm S.D. of four separate experiments; $* P<0.05 ; * * P<0.01$; ***P $P 0.001$ vs. the zero concentration control; ${ }^{\text {,ff }} P<0.01$; fff $P<0.001$; ${ }^{\xi \xi \xi} P<0.001$ vs. the indicated groups.

$50 \mu \mathrm{g} / \mathrm{ml}$ concentrations of soy PG blocked the increase in IL1 $\beta$, TLR1, and TLR2. However, soy PG at the higher dose actually increased LPS-induced IL1 $\alpha$ and TNF $\alpha$ inflammatory mediator expression. This result suggests that soy PG can inhibit some of the inflammatory mediators that are expressed upon the activation of TLR4 but that soy PG itself might be slightly proinflammatory or irritating, suggesting that this PG mixture could cause skin inflammation and therefore might not be ideal for treatment of dermatological disorders.

Effects of Soy PG on the Keratinocyte Expression of Inflammatory Mediators upon Stimulation with Recombinant S100A9. We have previously shown that S100A9 acts as a DAMP to activate TLR2 and TLR4 and induce keratinocyte inflammatory mediator production and that DOPG blocks the activation of both pattern recognition receptors (Choudhary et al., 2019). In addition, in prior findings, we determined that soy PG inhibits inflammation induced by a contact irritant (Xie et al., 2018), and we postulated that this inhibition might relate to an ability of soy PG to block inflammatory mediator production in response to contact irritant-induced protein expression of DAMPs such as S100A9. To examine whether the inhibitory effect on DAMP-stimulated inflammatory mediator induction was specific to DOPG and/or whether the inhibition of inflammation by soy PG could involve its blocking of DAMP-induced inflammatory mediator production, we determined the effect of soy PG on S100A9-induced inflammatory mediator expression in keratinocytes. We observed that, as with DOPG (Choudhary et al., 2019), soy PG inhibited S100A9-induced IL1 $\beta$ and IL6 as well as TLR2 and TLR4 expression (Fig. 5). On the other hand, soy PG at a concentration of $10 \mu \mathrm{g} / \mathrm{ml}$ slightly but statistically significantly increased TNF $\alpha$ mRNA levels, and $50 \mu \mathrm{g} / \mathrm{ml}$ soy PG markedly elevated expression, with no additional stimulatory or inhibitory effect with S100A9. The fact that soy PG alone exhibits some inflammatory effects again suggests that another PG species may be a better choice for use as a treatment for inflammation associated with infection or skin diseases such as psoriasis.

Effects of DOPG on the $\mathrm{Pam}_{3} \mathrm{CSK}_{4}$-Induced Keratinocyte Expression of Inflammatory Mediators. Soy PG has a fatty acid composition that includes palmitic acid (16:0; $17 \%)$, stearic acid (18:0; 6\%), oleic acid (18:1; 13\%), linoleic acid $(18: 2 ; 59 \%)$, and linolenic acid (18:3; 5\%). Soy PG was selected in our previous experiment (Xie et al., 2018) because PG species composed of polyunsaturated fatty acids were most 
A

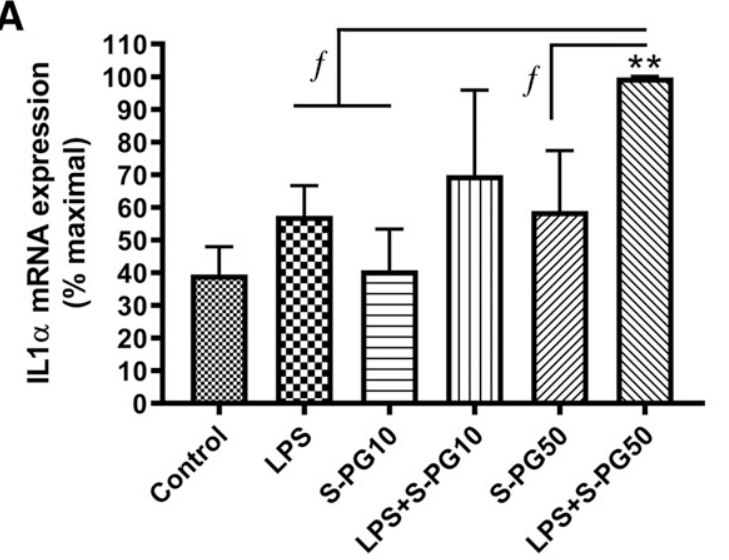

C

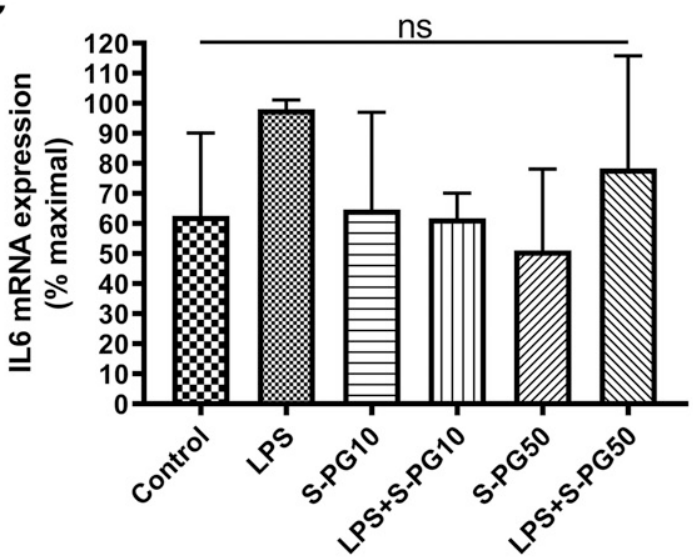

E

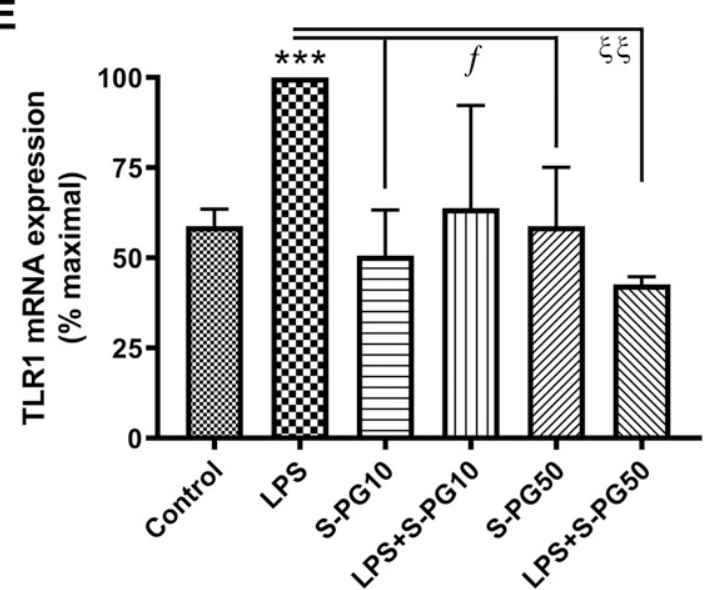

B

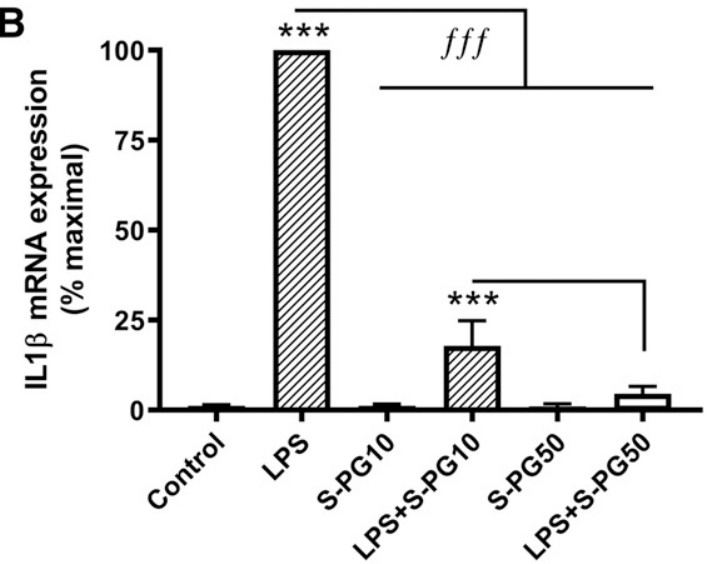

D

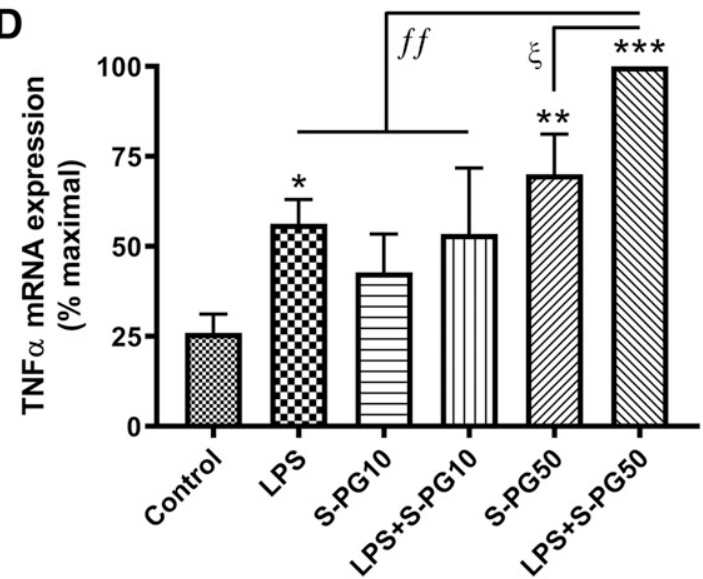

$\mathbf{F}$

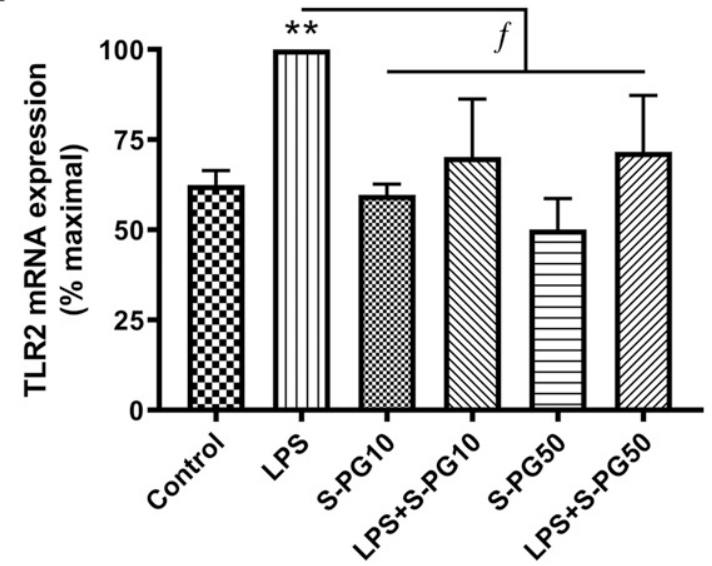

Fig. 4. Soy $P G$ inhibited keratinocyte inflammatory mediator expression in response to LPS. Keratinocytes were treated with 0 or $1000 \mathrm{U} / \mathrm{ml} L P S$ in the presence or absence of 10 or $50 \mu \mathrm{g} / \mathrm{ml}$ soy PG (s-PG) for 2 hours. RNA was then isolated, and the expression of the inflammatory mediators (A) IL1 $\alpha$, (B) IL1 $\beta$, (C) IL6, and (D) TNF $\alpha$ as well as (E) TLR1 and (F) TLR2 was monitored by quantitative RT-PCR with GAPDH used as the housekeeping gene. Results represent the means \pm S.D. of three separate experiments; $* P<0.05 ; * * P<0.01 ; * * * P<0.001$ vs. the zero concentration control; ${ }^{\text {f }} P<0.05 ;{ }^{\text {ff }} P<$ $0.01 ;{ }^{\mathrm{fff}} P<0.001 ;{ }^{\xi} P<0.05 ;{ }^{\xi \xi} P<0.01$ vs. the indicated groups; ns, not significantly different.

efficacious in inhibiting keratinocyte proliferation (Xie et al., 2014). To determine if the ability of PG to inhibit TLR2induced inflammatory mediators was unique to its high proportion of polyunsaturated fatty acids, we also examined the effect of DOPG on $\mathrm{Pam}_{3} \mathrm{CSK}_{4}$-elicited inflammatory mediator expression. As shown in Fig. 6, both concentrations of DOPG (50 and $100 \mu \mathrm{g} / \mathrm{ml})$ statistically significantly inhibited the $\mathrm{Pam}_{3} \mathrm{CSK}_{4}$-induced increase in the expression of all cytokines tested. Thus, DOPG produced similar, but not identical, effects on the TLR1/2-induced cytokine expression as soy $\mathrm{PG}$.

Effects of DOPG on the $\mathrm{Pam}_{3} \mathrm{CSK}_{4}$-Induced Expression of Inflammatory Mediators in a RAW264.7 Macrophage Cell Line. Keratinocytes and inflammatory mediators released by these cells are likely involved in inflammatory skin diseases such as psoriasis; nevertheless, inflammation is thought to be predominantly mediated by the immune system. Therefore, we investigated the ability of $\mathrm{PG}$ to inhibit inflammatory 
A
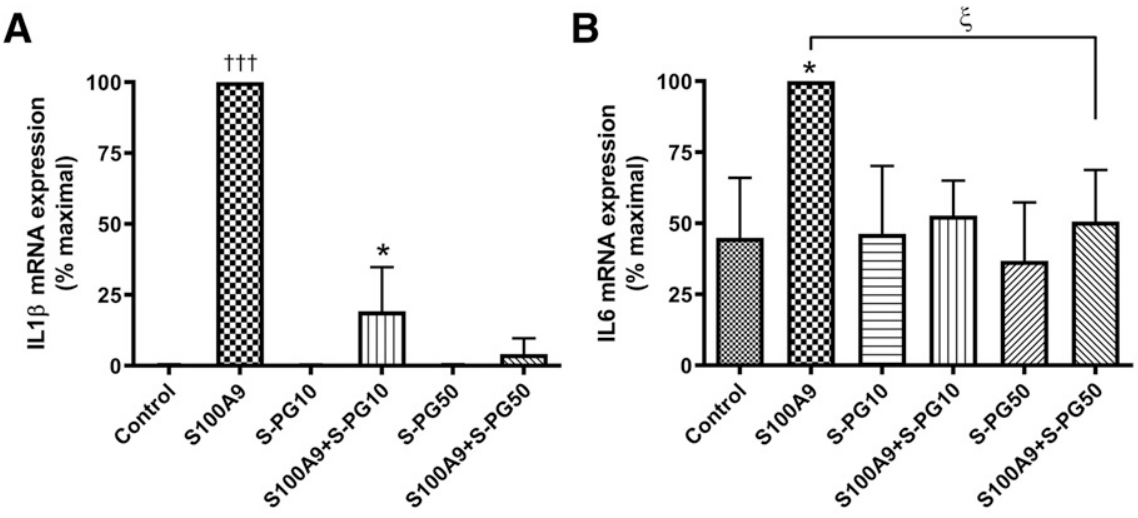

$E$

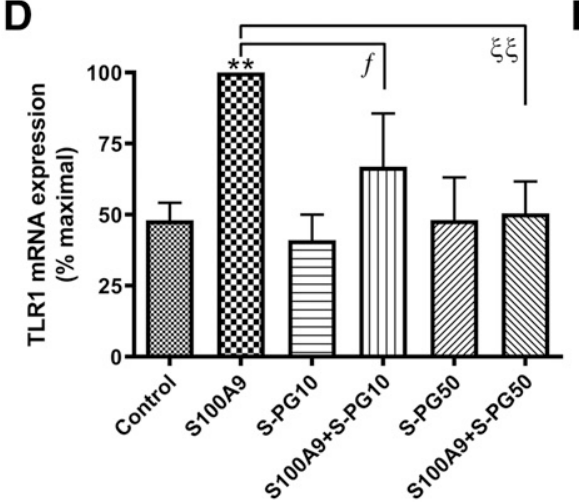

B

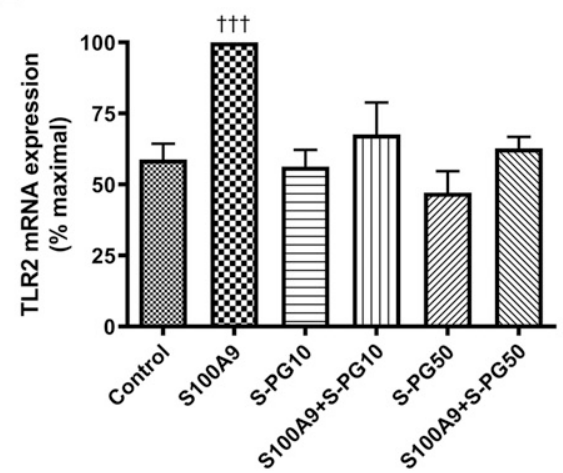

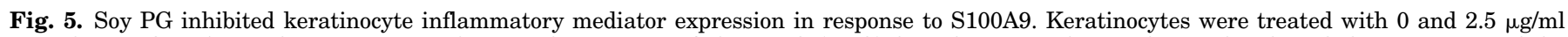

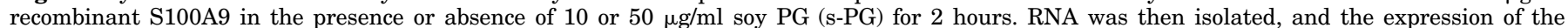

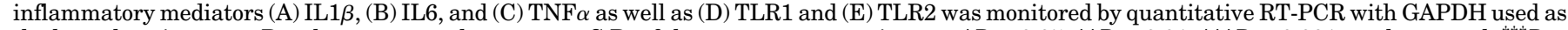

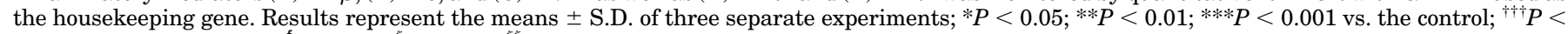
0.001 vs. all other groups; ${ }^{f} P<0.05 ;{ }^{\xi} P<0.05 ;{ }^{\xi \xi} P<0.01$ vs. the indicated groups.

mediator expression in the cell line RAW264.7, which serves as a model for macrophages, an immune cell. Similar to keratinocytes, upon stimulation with $\mathrm{Pam}_{3} \mathrm{CSK}_{4}$, RAW264.7 cells also upregulated their mRNA levels of IL $1 \alpha$, IL $1 \beta$, IL6, and TNF $\alpha$ (Fig. 7). Cotreatment with DOPG $(100 \mu \mathrm{g} / \mathrm{ml})$ resulted in an inhibition of $\mathrm{Pam}_{3} \mathrm{CSK}_{4}$ 's induction of IL1 $\alpha$, IL1 $\beta$, and IL6; the lower $(50 \mu \mathrm{g} / \mathrm{ml})$ DOPG concentration also inhibited IL $1 \alpha$ and $-\beta$ expression. Consistent with this result, we also found, as illustrated in Fig. 8, A and B, that DOPG inhibited the phosphorylation (activation) of $\mathrm{NF}_{\kappa} \mathrm{B}$. In addition, DOPG, but not the control lipid DOPC, inhibited $\mathrm{Pam}_{3} \mathrm{CSK}_{4}$-induced nuclear translocation (i.e., activation) of $\mathrm{NF}_{\kappa} \mathrm{B}$ (Fig. 8C), a transcription factor downstream of pattern recognition receptors such as TLR2 and TLR4.

The effect of DOPG on $\mathrm{NF}_{\kappa} \mathrm{B}$ activation in response to the TLR7/8 agonist Resiquimod was also examined. As illustrated in Fig. 9, Resiquimod, like $\mathrm{Pam}_{3} \mathrm{CSK}_{4}$, increased the levels of phosphorylated (activated) $\mathrm{NF} \kappa \mathrm{B}$. However, in contrast to the TLR2 agonist and consistent with its minimal effects on Resiquimod-induced inflammatory mediator expression (Choudhary et al., 2019), DOPG did not affect Resiquimodinduced $\mathrm{NF}_{\kappa} \mathrm{B}$ activation, suggesting that DOPG was not a general inhibitor of TLR and/or NF $\kappa \mathrm{B}$ activation.

DOPG-Mediated Inhibition of TLR2 and TLR4 Activation Occurs in Human Cells Expressing These Pattern-Recognition Receptors. We next sought to extend our findings in mouse keratinocytes and macrophages to human cells expressing these receptors. To do so, we made use of two reporter cell lines, one that overexpresses and reports on human TLR2 and the other that overexpresses and reports on human TLR4. We first validated these reporter cell lines, demonstrating a dose-dependent increase in reporter activity with $\mathrm{Pam}_{3} \mathrm{CSK}_{4}$ in the hTLR2 and LPS in the hTLR4 cell lines (Fig. 10, A and B). We then showed that DOPG markedly inhibited reporter activity in both cell lines, whereas DOPC, as a negative control, exerted a slight (but statistically significant) inhibition of TLR2, but not TLR4, activity (Fig. 10, C and D).

\section{Discussion}

We have previously shown in primary mouse keratinocytes that AQP3 and PLD2 are associated (physically and functionally) and that this association allows for the generation of $\mathrm{PG}$, a lipid second messenger involved in regulating keratinocyte proliferation and differentiation (reviewed in Qin et al., 2011). PG comprises different species depending on its fatty acid composition, and our prior study has indicated that these different species can have differential effects on keratinocytes (Xie et al., 2014). This idea is consistent with findings in the lung demonstrating that unsaturated PG species inhibit surfactant protein A's anti-inflammatory effects on macrophages exposed to LPS but saturated PG species do not (Chiba et al., 2006). This differential PG molecular species effect might also underlie the unexpected finding that $\mathrm{Pam}_{3} \mathrm{CSK}_{4}$-induced 
A

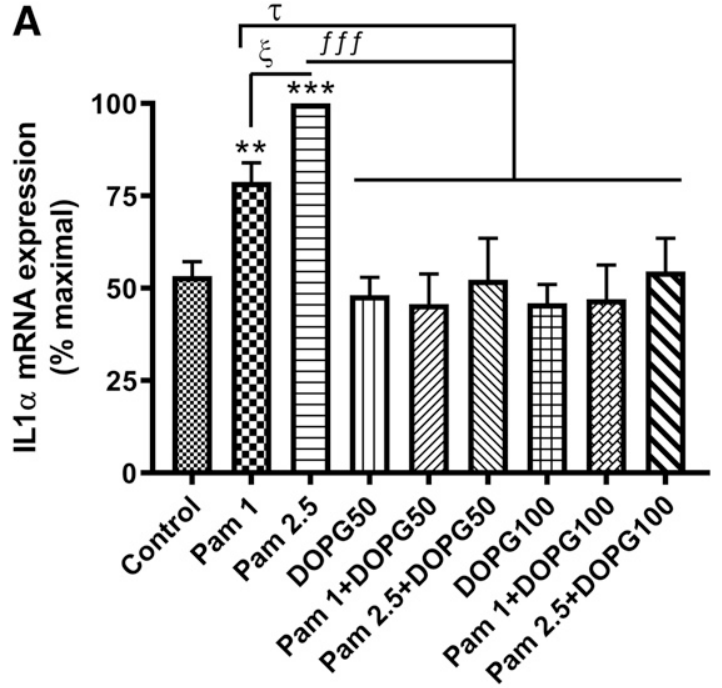

C

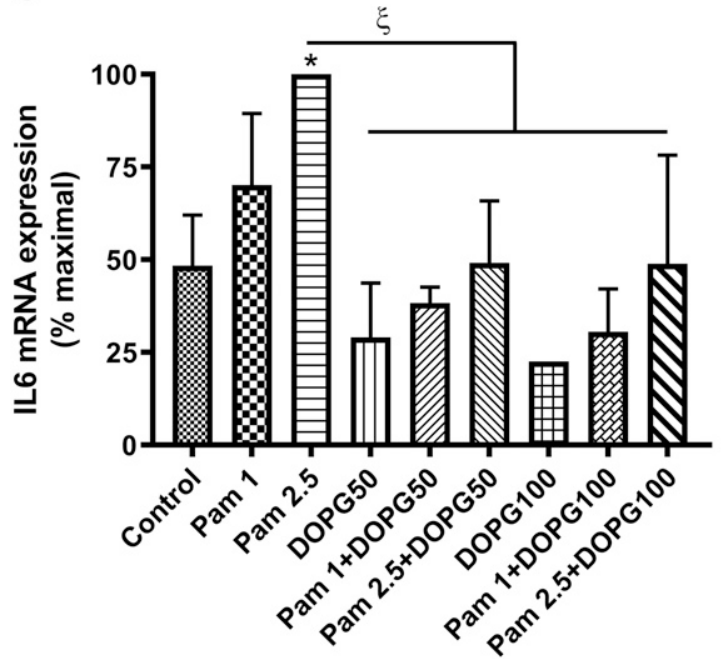

B

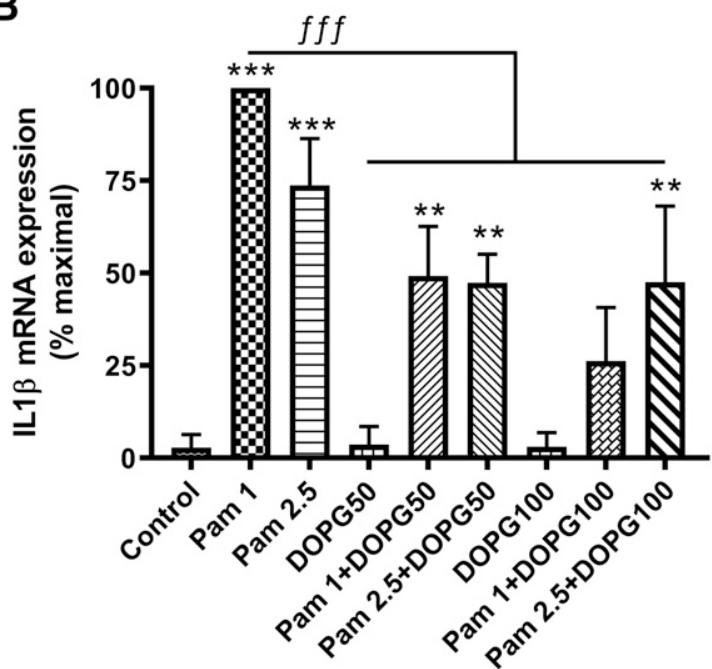

D

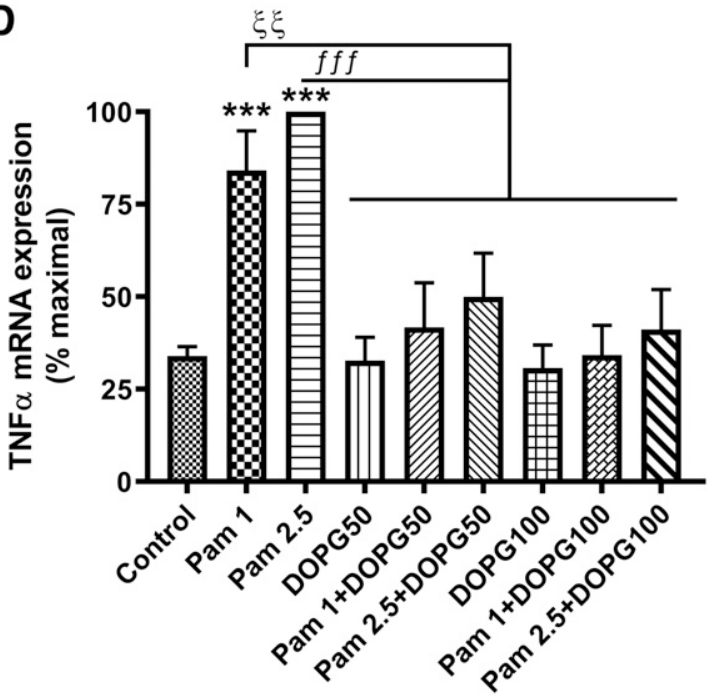

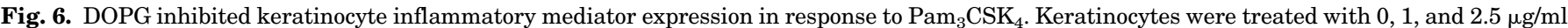

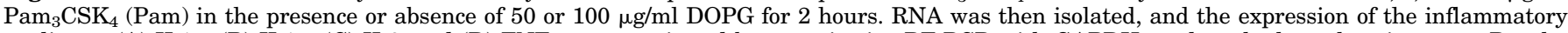

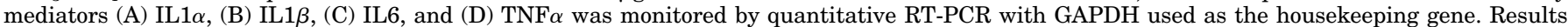

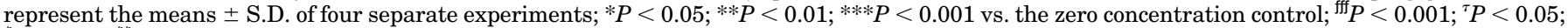
${ }^{\xi} P<0.05 ;{ }^{\xi \xi} P<0.01$ vs. the indicated groups.

IL1 $\beta$ expression was not always inhibited by PG; thus, in RAW264.7 cells, DOPG was unable to repress the induction of IL1 $\beta$ expression, although DOPG, but not soy PG, reduced IL1 $\beta$ expression in keratinocytes. This result suggests the possibility that the TLR2 agonist's ability to interact or not with its receptor is influenced by the fatty acid composition of the phospholipid, or perhaps it suggests more than one mode of induction of IL $1 \beta$, particularly in immune cells. In addition, it is known that multiple signal transduction pathways can crosstalk with and regulate TLR signaling and downstream $\mathrm{NF}_{\kappa} \mathrm{B}$, including mitogenactivated protein kinase, mammalian target of rapamycin, and those kinases activated by TNF and interferons as well as the endoplasmic reticulum stress-activated kinase, pancreatic eIF $2 \alpha$ endoplasmic reticulum kinase, and the nutrient deprivation-activated protein kinase general control nonderepressible-2 (reviewed in Adelaja and Hoffmann, 2019). Thus, differences in the activity of one or more of these other pathways in the two cell types might result in
PG's differential repression of IL $1 \beta$ expression in response to the TLR2 agonist.

We previously found that synthetic polyunsaturated fatty acid-containing PGs showed the greatest efficacy to inhibit keratinocyte proliferation in vitro (Xie et al., 2014); however, synthetic PGs may be costly to produce, possibly impeding their development as a psoriasis therapy. On the other hand, soy $\mathrm{PG}$ is relatively inexpensive to produce and is also a natural product, suggesting possible advantages to its use as a treatment of psoriasis. Thus, in a previous study, we selected soy PG, which is a mixture of PG species with a high percentage of polyunsaturated fatty acids, to act as a potential anti-inflammatory agent and found that soy PG did in fact inhibit inflammation in a contact irritant mouse model in vivo (Xie et al., 2018). In the present study, we investigated the potential mechanism of soy PG's anti-inflammatory properties. Our results indicated that keratinocytes responded to TLR2 and TLR4 agonists with inflammatory mediator expression and that soy PG inhibited TLR2 and TLR4 activation 
A

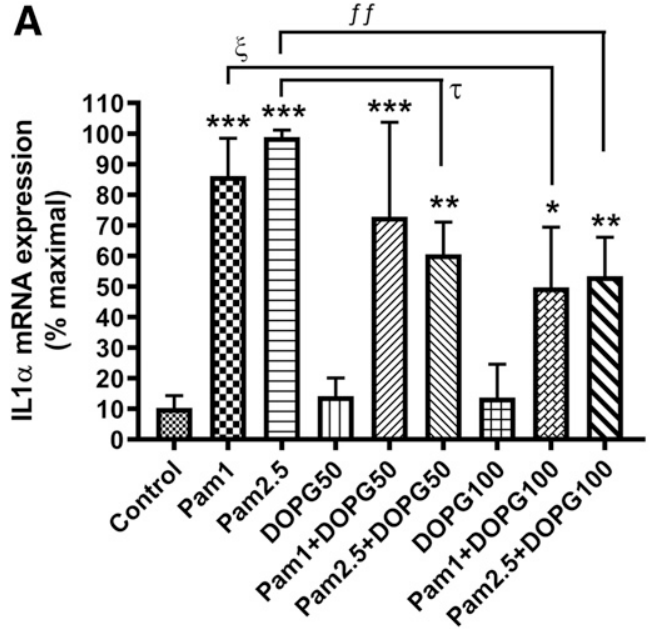

C

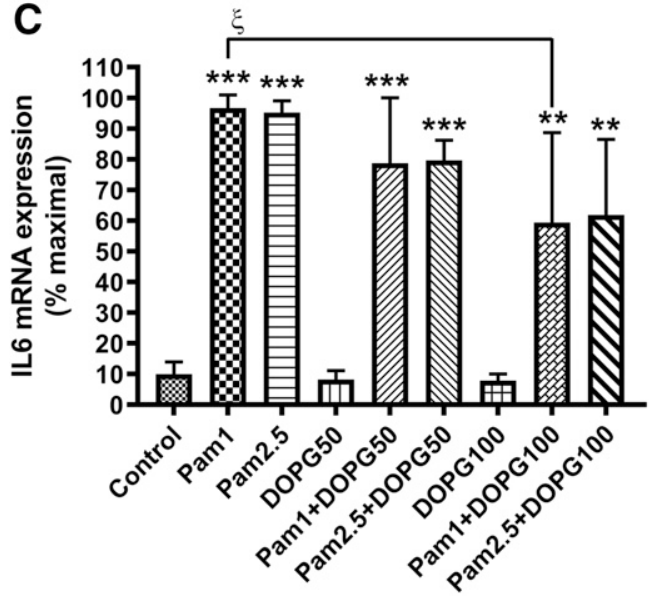

B
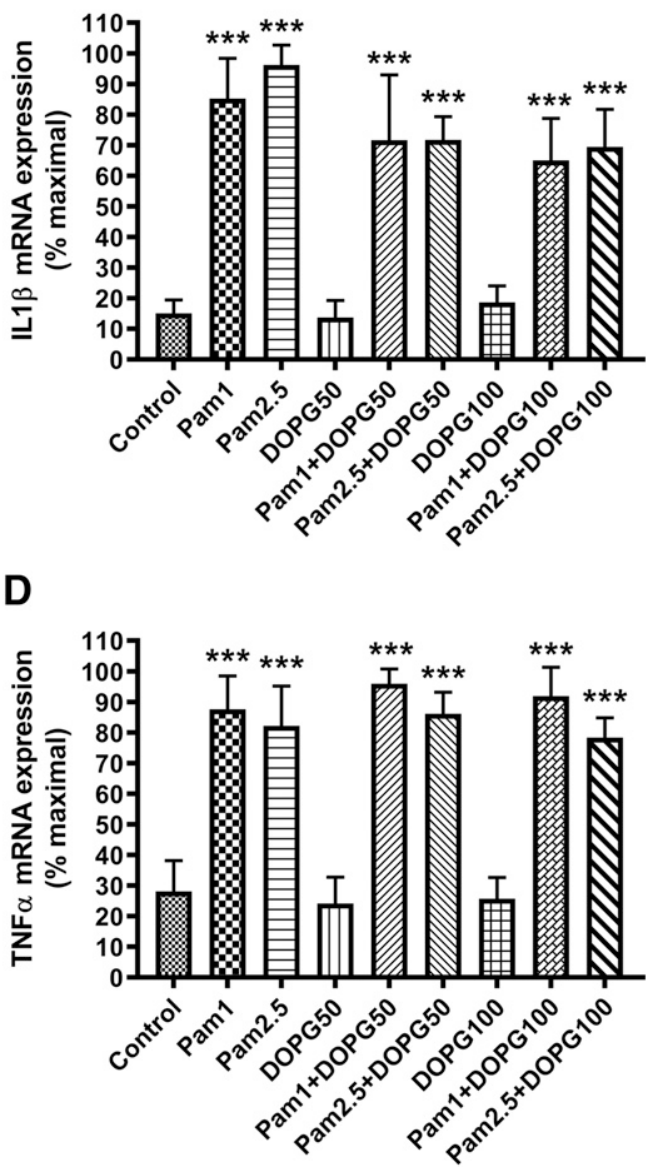

Fig. 7. DOPG inhibited $\mathrm{Pam}_{3} \mathrm{CSK}_{4}$-induced inflammatory mediator expression in the macrophage cell line RAW264.7. RAW264.7 cells were treated with 0,1 , and $2.5 \mu \mathrm{M} \mathrm{Pam}{ }_{3} \mathrm{CSK}_{4}(\mathrm{Pam})$ in the presence or absence of 50 or $100 \mu \mathrm{g} / \mathrm{ml}$ DOPG for 2 hours. RNA was then isolated, and the expression of the inflammatory mediators (A) IL1 $\alpha$, (B) IL1 $\beta$, (C) IL6, and (D) TNF $\alpha$ was monitored by quantitative RT-PCR with GAPDH used as the housekeeping gene. Results represent the means \pm S.D. of four separate experiments; $* P<0.05$; $* * P<0.01$; ${ }^{* * *} P<0.001$ vs. the zero concentration control; $f P<0.05$; ${ }^{*} P<$ $0.01 ;{ }^{\tau} P<0.05 ;{ }^{\xi} P<0.05$ vs. the indicated groups.

in both keratinocytes and a macrophage cell line. However, we observed that soy PG had some proinflammatory effects itself, particularly at higher concentrations.

The contact irritant, 12-O-tetradecanoylphorbol 13-acetate, used in our previous study is known to induce the expression of S100A8 and S100A9 as well as various inflammatory cytokines in keratinocytes (Carlsson et al., 2005; Helwa et al., 2015) and skin (Gebhardt et al., 2002; reviewed in Mueller, 2006). Based on the results presented here, it seems possible that soy PG may inhibit inflammation in this ear edema model at least in part by blocking TLR activation by DAMPs such as S100A9 (Choudhary et al., 2019) as well as PAMPs expressed by the skin microbiome. Our results showing that in keratinocytes, soy PG inhibits DAMP-elicited expression of TLR1/2 (Fig. 5), which in addition to di- and triacylated microbial peptides responds to DAMPs such as S100A9 and $\beta$-defensin that are also upregulated in psoriasis (Nakajima et al., 2011; Racz et al., 2011; Waite and Skokos, 2012; Choudhary et al., 2019), suggests the potential utility of soy PG for the treatment of inflammation in psoriasis. Similarly, its repression of IL6, for which serum levels are markedly elevated in psoriasis patients in comparison with healthy controls (Arican et al., 2005), provides additional support for the use of soy PG to suppress skin inflammation. However, as noted above, our results demonstrating that soy $\mathrm{PG}$ induces $\mathrm{TNF} \alpha$ expression are problematic, as TNF $\alpha$ levels are elevated in psoriasis, with drugs targeting the $\mathrm{TNF} \alpha$ pathway showing efficacy for treatment of the disease (reviewed in Brotas et al., 2012). Thus, our findings suggest that soy PG exhibits a narrow therapeutic window, and despite its ability to inhibit the proliferation of rapidly dividing keratinocytes, soy PG would likely not be practicable as a therapy to treat inflammatory skin diseases such as psoriasis.

Both soy PG and DOPG reduced the TLR1/2-elicited mRNA levels of IL $1 \alpha$, IL6, and TNF $\alpha$ in keratinocytes in response to $\mathrm{Pam}_{3} \mathrm{CSK}_{4}$. Soy PG appeared to be quite efficacious, as a concentration as low as $10 \mu \mathrm{g} / \mathrm{ml}$ produced a statistically significant inhibition of IL6 and TNF $\alpha$ mRNA levels. It should be noted that the effect of soy PG and DOPG on the inflammatory mediator profile was similar but not identical, with soy PG exerting little effect on IL1 $\beta$, whereas DOPG inhibited the $\mathrm{Pam}_{3} \mathrm{CSK}_{4}$-induced keratinocyte expression of all of the tested inflammatory mediators. In contrast to keratinocytes, in the RAW264.7 macrophage cell line, DOPG did not reduce the effect of $\mathrm{Pam}_{3} \mathrm{CSK}_{4}$ on IL1 $\beta$ or TNF $\alpha$ expression, although it inhibited the $\mathrm{Pam}_{3} \mathrm{CSK}_{4}$-induced 
A

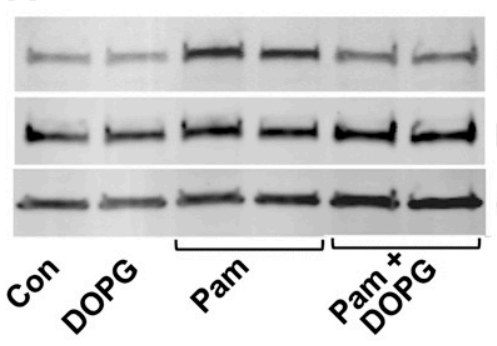

C
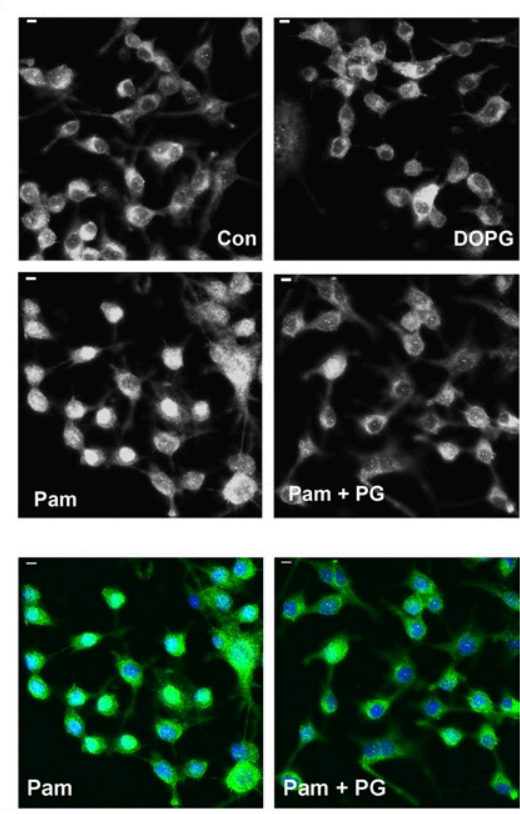

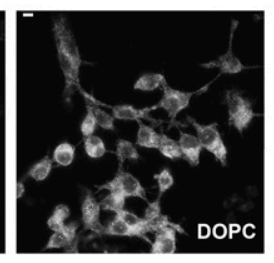

B
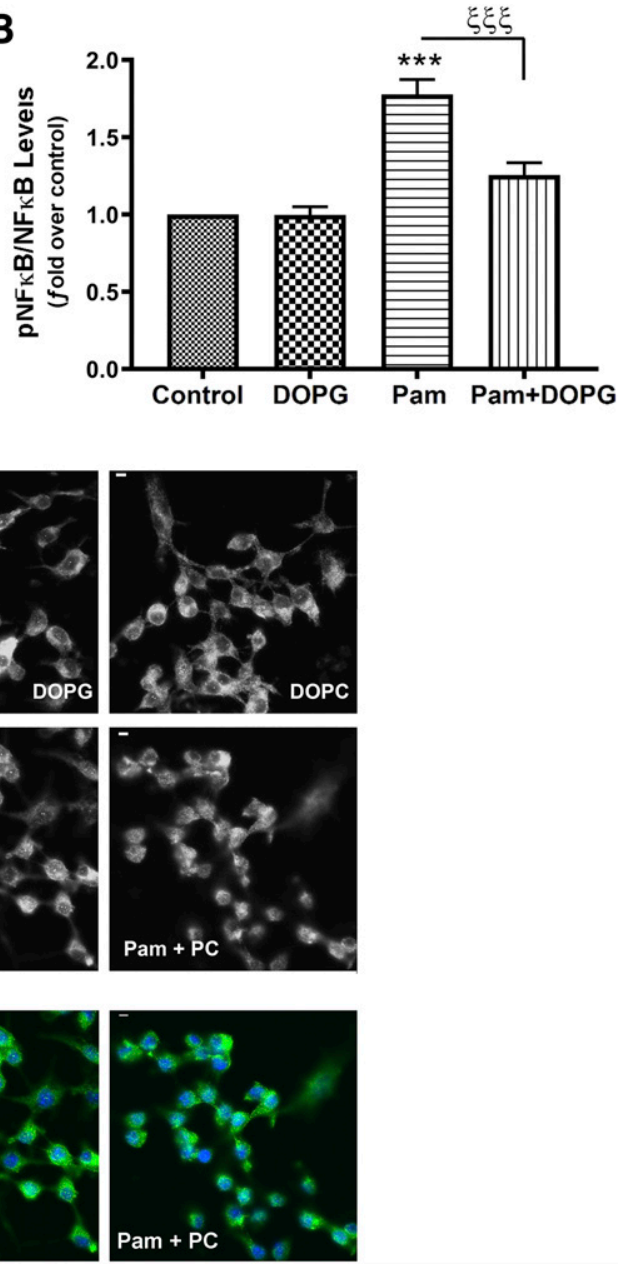

Fig. 8. DOPG inhibited $\mathrm{Pam}_{3} \mathrm{CSK}_{4}{ }^{-}$ induced $\mathrm{NF}_{\kappa} \mathrm{B}$ activation in the macrophage cell line RAW264.7. (A) RAW264.7 cells were treated with or without $2.5 \mu \mathrm{g} /$ $\mathrm{ml} \mathrm{Pam}{ }_{3} \mathrm{CSK}_{4}(\mathrm{Pam})$ in the presence and absence of $100 \mu \mathrm{g} / \mathrm{ml}$ DOPG for 10 minutes. Cells were harvested and the phosphorylation (activation) status of $\mathrm{NF}_{\kappa} \mathrm{B}$ determined using an antibody recognizing phosphoserine ${ }^{536}$ in comparison with total NF $\kappa$ B levels. (A) shows a representative Western blot, whereas (B) presents the cumulative results from three separate experiments (means \pm S.D.); $* * * P<0.001$ vs. the control; $\xi \xi \xi P<0.001$ as indicated. (C) RAW264.7 cells were treated with 0 and $2.5 \mu \mathrm{M} \mathrm{Pam}{ }_{3} \mathrm{CSK}_{4}$ (Pam) in the presence or absence of $100 \mu \mathrm{g} / \mathrm{ml}$ DOPG or DOPC as indicated for 2 hours. $\mathrm{NF}_{\kappa} \mathrm{B}$ was then monitored by immunocytochemistry and confocal microscopy (Scale bar, $5 \mu \mathrm{m}$ ). The final row shows the same micrographs illustrating grayscale $\mathrm{NF}_{\kappa} \mathrm{B}$ staining as in the row above, with $\mathrm{NF}_{\kappa} \mathrm{B}$ staining in green and nuclear staining (with DAPI) in blue in these merged color images. Results are representative of at least three separate experiments. increase in mRNA levels of the other two inflammatory mediators. Together, these results argue against a nonspecific effect of PG to inhibit $\mathrm{Pam}_{3} \mathrm{CSK}_{4}$ 's action, an idea that is further confirmed by the inability of another lipid (DOPC) to block TLR2 or TLR4 activation. The lack of effect of PG alone on the expression of several of the inflammatory mediators also suggests that the inhibitory effects of $P G$ are not the result of cytotoxicity of this phospholipid. DOPG also did not exhibit a general inhibition of all TLRs, as it inhibited neither $\mathrm{NF} \kappa \mathrm{B}$ activation (Fig. 9) nor inflammatory mediator expression (Choudhary et al., 2019) induced by the TLR7/8 agonist
Resiquimod. Furthermore, our results indicated that DOPG had a broad therapeutic range for the inhibition of TLR2 and TLR4 activation by $\mathrm{Pam}_{3} \mathrm{CSK}_{4}$ and LPS (this study) and endogenous molecules that engage these pattern-recognition receptors (Choudhary et al., 2019), with limited or no ability to induce inflammatory mediator production on its own. Thus, our results provide evidence for the potential use of DOPG to treat inflammatory skin disorders such as psoriasis.

A comparison of the results observed in keratinocytes with those in the lung and bronchial epithelial cells is also in order. In general, Kandasamy et al. (2011) have demonstrated an effect of
A

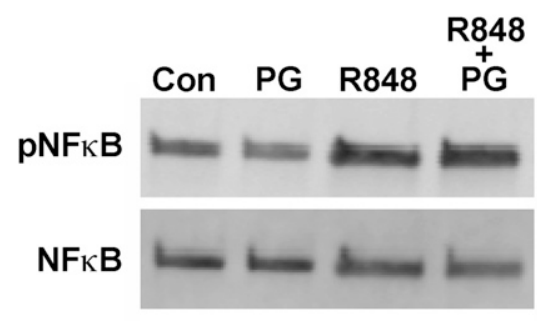

B

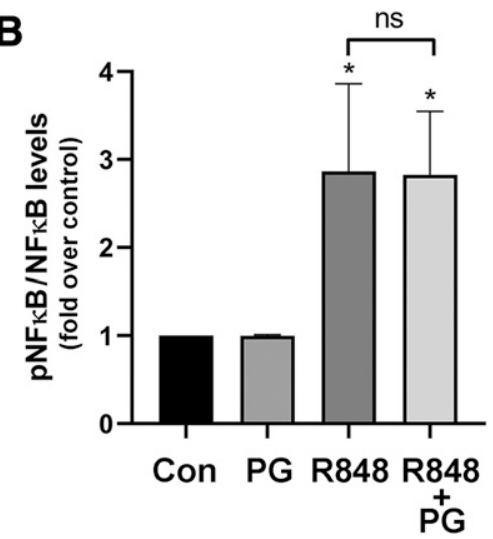

Fig. 9. DOPG had no effect on R848-induced $\mathrm{NF}_{\kappa} \mathrm{B}$ activation in the macrophage cell line RAW264.7. (A) RAW264.7 cells were treated with or without $1 \mu \mathrm{g} / \mathrm{ml}$ R848 (Resiquimod) in the presence and absence of 100 $\mu \mathrm{g} / \mathrm{ml}$ DOPG for 10 minutes. Cells were harvested and the phosphorylation (activation) status of $\mathrm{NF}_{\kappa} \mathrm{B}$ determined using an antibody recognizing phosphoserine ${ }^{536}$ in comparison with total NF $\kappa$ B levels. (A) shows a representative Western blot, whereas (B) presents the cumulative results from three separate experiments (means \pm S.D.); $* P<0.05$ vs. the control; ns, not significantly different. 
A

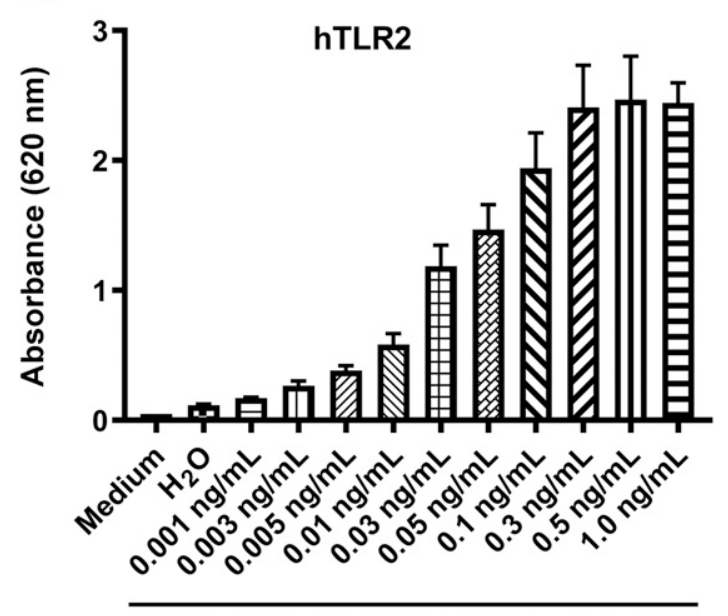

$\mathrm{Pam}_{3} \mathrm{CSK}_{4}$

C

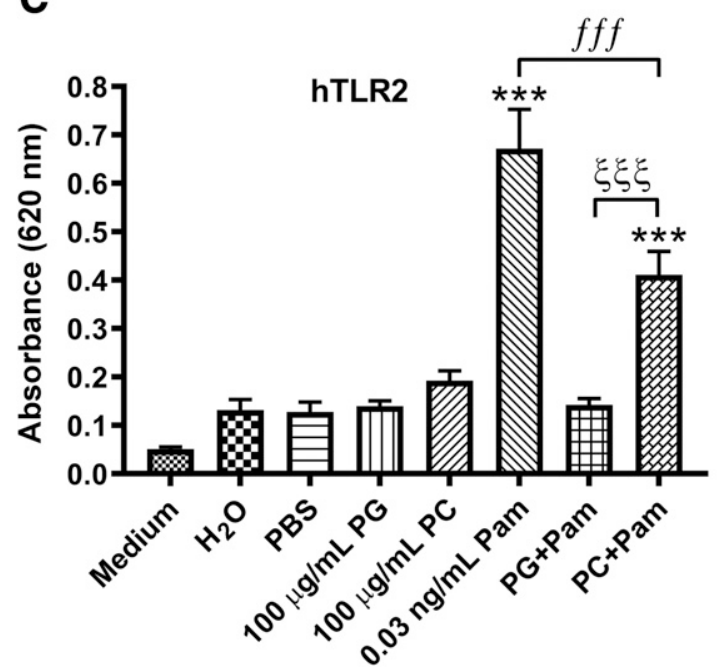

B

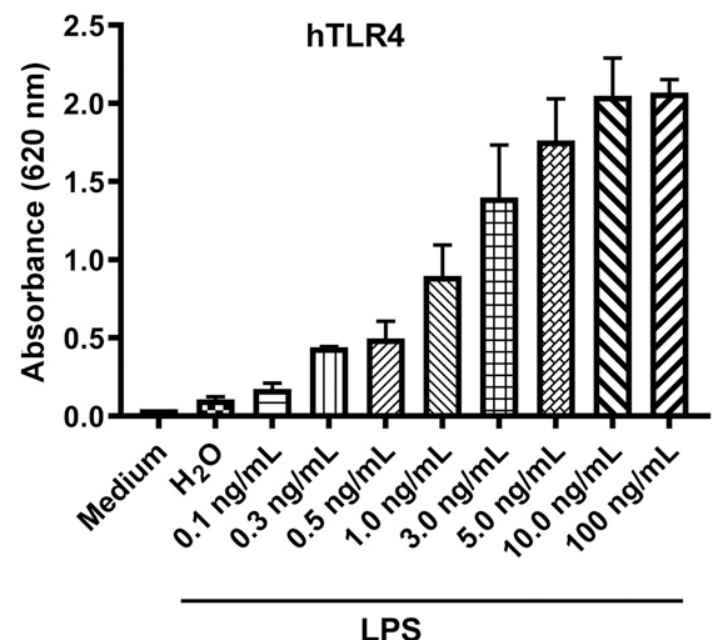

D

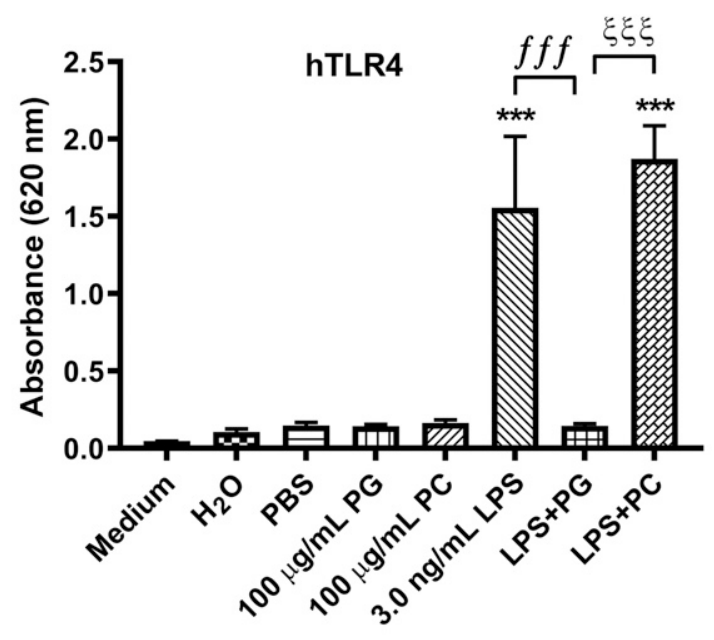

Fig. 10. DOPG, but not DOPC, inhibited $\mathrm{Pam}_{3} \mathrm{CSK}_{4}$ - and LPS-induced TLR2 and TLR4 activation in reporter cell lines. (A) HEK-Blue-hTLR2 cells were incubated for 24 hours in the HEK-Blue detection medium with various concentrations of $\mathrm{Pam}_{3} \mathrm{CSK}_{4}$, as indicated. (B) HEK-Blue-hTLR4 cells were incubated for 24 hours in the HEK-Blue detection medium with various concentrations of LPS, as indicated. (C) HEK-Blue-hTLR2 cells were incubated in the HEK-Blue detection medium with or without $0.03 \mathrm{ng} / \mathrm{ml} \mathrm{Pam}_{3} \mathrm{CSK}_{4}$ in the presence and absence of $100 \mu \mathrm{g} / \mathrm{ml}$ DOPG or DOPC for $24 \mathrm{hours}$. (D) HEKBlue-hTLR4 cells were incubated in the HEK-Blue detection medium with or without $3 \mathrm{ng} / \mathrm{ml}$ LPS in the presence and absence of $100 \mu \mathrm{g} / \mathrm{ml}$ DOPG or DOPC for 24 hours. In all cases, SEAP activity was then measured as absorbance at $620 \mathrm{~nm}$. Values represent the means \pm S.D. from at least three separate experiments; *** $P<0.001$ vs. the control; fff $P<0.001 ;{ }^{\xi \xi \xi} P<0.001$ as indicated.

palmitoyl-oleoylphosphatidylglycerol to reduce TLR2-mediated cellular responses. Thus, palmitoyl-oleoylphosphatidylglycerol inhibits TLR2-mediated arachidonic acid release from mouse and human macrophages exposed to Mycoplasma pneumonia membrane despite having no effect on Mycoplasma binding to the cell surface (Kandasamy et al., 2011). In these experiments, DOPG induces a small but not statistically significant decrease in arachidonic acid release. Nevertheless, these authors have also shown that DOPG is most potent in terms of inhibition of IL8 production in BEAS2B human bronchial epithelial cells (Numata et al., 2013). Consistent with this result, DOPG has been found in macrophages to inhibit endotoxin-stimulated $\mathrm{NF}_{\kappa} \mathrm{B}$ activation, thereby reducing Type IIA secretory phospholipase $\mathrm{A}_{2}$ levels and activity (Wu et al., 2003), although these authors did not investigate other PG species.

Finally, the molecular mechanism by which PG inhibits TLR2/4 activation is reported to be through the protein CD14.
Thus, Kuronuma et al. (2009) have shown that PG binds to MD2 and CD14, an accessory protein and a coreceptor, respectively, for TLR2 and TLR4 (Chun and Seong, 2010; van Bergenhenegouwen et al., 2013; He et al., 2016). Subsequent studies showed that the ability of PG to inhibit TLR2 and TLR4 activation corresponded with its interaction with CD14 rather than MD2 (Kandasamy et al., 2016). PG inhibits the binding of di[3-deoxy-D-manno-octulosonyl]-lipid A (a recombinant LPS analog) to CD14 and likely interferes with CD14's ability to present such microbial components to the appropriate TLR (Kandasamy et al., 2016), but further investigation is required.

In conclusion, this study shows for the first time to our knowledge that soy PG suppressed TLR2 and TLR4 activation and induction of inflammatory mediator expression by microbial components and other PAMPs in keratinocytes. In addition, the demonstrated ability of TLR2 activation to 
induce S100 proteins, and TLR4 activation to stimulate TLR1/ 2 expression, may initiate a positive feedback loop in keratinocytes that promotes and sustains skin inflammation in inflammatory skin diseases such as psoriasis. Thus, our results suggest that $\mathrm{PG}$, particularly DOPG, may be effective for the treatment of skin diseases characterized by inflammation.

\section{Acknowledgments}

We are grateful for the expert technical assistance of Purnima Merai and for the kind gift of RAW264.7 cells from Drs. Zhong and Isales.

\section{Authorship Contributions}

Participated in research design: Choudhary, Chen, Bollag.

Conducted experiments: Choudhary, Griffith, Chen.

Performed data analysis: Choudhary, Griffith, Chen.

Wrote or contributed to the writing of the manuscript: Choudhary, Griffith, Chen, Bollag.

\section{References}

Adelaja A and Hoffmann A (2019) Signaling crosstalk mechanisms that may finetune pathogen-responsive NFкB. Front Immunol 10:433.

Arican O, Aral M, Sasmaz S, and Ciragil P (2005) Serum levels of TNF-alpha, IFNgamma, IL-6, IL-8, IL-12, IL-17, and IL-18 in patients with active psoriasis and correlation with disease severity. Mediators Inflamm 2005:273-279.

Bollag WB, Ducote J, and Harmon CS (1993) Effects of the selective protein kinase C inhibitor, Ro 31-7549, on the proliferation of cultured mouse epidermal keratinocytes. J Invest Dermatol 100:240-246.

Bollag WB, Xie D, Zhong X, and Zheng X (2007) A potential role for the phospholipase D2-aquaporin-3 signaling module in early keratinocyte differentiation: production of a phosphatidylglycerol signaling lipid. J Invest Dermatol 127:2823-2831.

Brotas AM, Cunha JM, Lago EH, Machado CC, and Carneiro SC (2012) Tumor necrosis factor-alpha and the cytokine network in psoriasis. An Bras Dermatol 87 673-681-683.

Carlsson H, Yhr M, Petersson S, Collins N, Polyak K, and Enerbäck C (2005) Psoriasin (S100A7) and calgranulin-B (S100A9) induction is dependent on reactive oxygen species and is downregulated by Bcl-2 and antioxidants. Cancer Biol Ther 4:998-1005.

Chiba H, Piboonpocanun S, Mitsuzawa H, Kuronuma K, Murphy RC, and Voelker DR (2006) Pulmonary surfactant proteins and lipids as modulators of inflammation and innate immunity. Respirology 11 (Suppl):S2-S6.

Choudhary V, Olala LO, Qin H, Helwa I, Pan ZQ, Tsai YY, Frohman MA, KaddourDjebbar I, and Bollag WB (2015) Aquaporin-3 re-expression induces differentiation in a phospholipase D2-dependent manner in aquaporin-3-knockout mouse keratinocytes. J Invest Dermatol 135:499-507.

Choudhary V, Uaratanawong R, Patel RR, Patel H, Bao W, Hartney B, Cohen E, Chen X, Zhong Q, Isales CM, et al. (2019) Phosphatidylglycerol inhibits toll-like receptor-mediated inflammation by danger-associated molecular patterns. J Invest Dermatol 139:868-877.

Chun KH and Seong SY (2010) CD14 but not MD2 transmit signals from DAMP. Int Immunopharmacol 10:98-106.

Gebhardt C, Breitenbach U, Tuckermann JP, Dittrich BT, Richter KH, and Angel P (2002) Calgranulins S100A8 and S100A9 are negatively regulated by glucocorticoids in a c-Fos-dependent manner and overexpressed throughout skin carcinogenesis. Oncogene 21:4266-4276.

Goldsmith LA (1991) Physiology, Biochemistry, and Molecular Biology of the Skin Oxford University Press, New York.

Griner RD, Qin F, Jung E, Sue-Ling CK, Crawford KB, Mann-Blakeney R, Bollag RJ, and Bollag WB (1999) 1,25-Dihydroxyvitamin D3 induces phospholipase D-1 expression in primary mouse epidermal keratinocytes. J Biol Chem 274:4663-4670.

He Z, Riva M, Björk P, Swärd K, Mörgelin M, Leanderson T, and Ivars F (2016) CD14 is a co-receptor for TLR4 in the S100A9-induced pro-inflammatory response in monocytes. PLoS One 11:e156377.

Helwa I, Patel R, Karempelis P, Kaddour-Djebbar I, Choudhary V, and Bollag WB (2015) The antipsoriatic agent monomethylfumarate has antiproliferative, prodifferentiative, and anti-inflammatory effects on keratinocytes. $J$ Pharmacol Exp Ther 352:90-97.

Kandasamy P, Numata M, Berry KZ, Fickes R, Leslie CC, Murphy RC, and Voelker DR (2016) Structural analogs of pulmonary surfactant phosphatidylglycerol inhibit toll-like receptor 2 and 4 signaling. J Lipid Res 57:993-1005.

Kandasamy P, Zarini S, Chan ED, Leslie CC, Murphy RC, and Voelker DR (2011) Pulmonary surfactant phosphatidylglycerol inhibits Mycoplasma pneumoniaestimulated eicosanoid production from human and mouse macrophages. $J$ Biol Chem 286:7841-7853.

Kuronuma K, Mitsuzawa H, Takeda K, Nishitani C, Chan ED, Kuroki Y, Nakamura M, and Voelker DR (2009) Anionic pulmonary surfactant phospholipids inhibit inflammatory responses from alveolar macrophages and U937 cells by binding the lipopolysaccharide-interacting proteins CD14 and MD-2. J Biol Chem 284: 25488-25500.

Langley R (2005) Psoriasis, Firefly Books, Inc., New York.

Mueller MM (2006) Inflammation in epithelial skin tumours: old stories and new ideas. Eur J Cancer 42:735-744.

Nakajima K, Kanda T, Takaishi M, Shiga T, Miyoshi K, Nakajima H, Kamijima R, Tarutani M, Benson JM, Elloso MM, et al. (2011) Distinct roles of IL-23 and IL-17 in the development of psoriasis-like lesions in a mouse model. $J$ Immunol 186: $4481-4489$

Numata M, Nagashima Y, Moore ML, Berry KZ, Chan M, Kandasamy P, Peebles RS Jr., Murphy RC, and Voelker DR (2013) Phosphatidylglycerol provides short-term prophylaxis against respiratory syncytial virus infection. $J$ Lipid Res 54: 2133-2143.

Pivarcsi A, Bodai L, Réthi B, Kenderessy-Szabó A, Koreck A, Széll M, Beer Z, BataCsörgoo Z, Magócsi M, Rajnavölgyi E, et al. (2003) Expression and function of Tolllike receptors 2 and 4 in human keratinocytes. Int Immunol 15:721-730.

Qin H, Zheng X, Zhong X, Shetty AK, Elias PM, and Bollag WB (2011) Aquaporin-3 in keratinocytes and skin: its role and interaction with phospholipase D2. Arch Biochem Biophys 508:138-143.

Rácz E, Prens EP, Kurek D, Kant M, de Ridder D, Mourits S, Baerveldt EM, Ozgur Z van IJcken WFJ, Laman JD, et al. (2011) Effective treatment of psoriasis with narrow-band UVB phototherapy is linked to suppression of the IFN and Th17 pathways. J Invest Dermatol 131:1547-1558.

Rapp SR, Feldman SR, Exum ML, Fleischer AB Jr., and Reboussin DM (1999) Psoriasis causes as much disability as other major medical diseases. J Am Acad Dermatol 41:401-407.

Schön MP (2019) Adaptive and innate immunity in psoriasis and other inflammatory disorders. Front Immunol 10:1764.

Stern RS, Nijsten T, Feldman SR, Margolis DJ, and Rolstad T (2004) Psoriasis is common, carries a substantial burden even when not extensive, and is associated with widespread treatment dissatisfaction. J Investig Dermatol Symp Proc 9: 136-139.

van Bergenhenegouwen J, Plantinga TS, Joosten LA, Netea MG, Folkerts G, Kraneveld AD, Garssen J, and Vos AP (2013) TLR2 \& Co: a critical analysis of the complex interactions between TLR2 and coreceptors. J Leukoc Biol 94:885-902.

Waite JC and Skokos D (2012) Th17 response and inflammatory autoimmune diseases. Int J Inflamm 2012:819467.

Wu YZ, Medjane S, Chabot S, Kubrusly FS, Raw I, Chignard M, and Touqui L (2003) Surfactant protein-A and phosphatidylglycerol suppress type IIA phospholipase A2 synthesis via nuclear factor-kappaB. Am J Respir Crit Care Med 168:692-699.

Xie D, Choudhary V, Seremwe M, Edwards JG, Wang A, Emmons AC, Bollag KA Johnson MH, and Bollag WB (2018) Soy phosphatidylglycerol reduces inflammation in a contact irritant ear edema mouse model in vivo. $J$ Pharmacol Exp Ther 366:1-8.

Xie D, Seremwe M, Edwards JG, Podolsky R, and Bollag WB (2014) Distinct effects of different phosphatidylglycerol species on mouse keratinocyte proliferation. PLoS One 9:e107119.

Yuspa SH, Hennings H, Tucker RW, Kilkenny A, Lee E, Kruszewski F, and Roop DR (1990) The Regulation of Differentiation in Normal and Neoplastic Keratinocytes, Wiley-Liss, Inc., New York.

Zheng X and Bollinger Bollag W (2003) Aquaporin 3 colocates with phospholipase d2 in caveolin-rich membrane microdomains and is downregulated upon keratinocyte differentiation. J Invest Dermatol 121:1487-1495.

Zheng X, Ray S, and Bollag WB (2003) Modulation of phospholipase D-mediated phosphatidylglycerol formation by differentiating agents in primary mouse epidermal keratinocytes. Biochim Biophys Acta 1643:25-36.

Address correspondence to: Wendy B. Bollag, Department of Physiology, Medical College of Georgia at Augusta University, 1120 15th St., Augusta, GA 30912. E-mail: wbollag@augusta.edu 